\title{
Accounting for Earnings Announcements in the Pricing of Equity Options
}

\author{
Tim Leung* Marco Santoli ${ }^{\dagger}$
}

September 4, 2018

\begin{abstract}
We study an option pricing framework that accounts for the price impact of an earnings announcement (EA), and analyze the behavior of the implied volatility surface prior to the event. On the announcement date, we incorporate a random jump to the stock price to represent the shock due to earnings. We consider different distributions of the scheduled earnings jump as well as different underlying stock price dynamics before and after the EA date. Our main contributions include analytical option pricing formulas when the underlying stock price follows the Kou model along with a double-exponential or Gaussian EA jump on the announcement date. Furthermore, we derive analytic bounds and asymptotics for the pre-EA implied volatility under various models. The calibration results demonstrate adequate fit of the entire implied volatility surface prior to an announcement. We also compare the risk-neutral distribution of the EA jump to its historical distribution. Finally, we discuss the valuation and exercise strategy of pre-EA American options, and illustrate an analytical approximation and numerical results.
\end{abstract}

Keywords: earnings announcement, equity options, pre-earnings announcement implied volatility

JEL Classification: G12, G13, G14

\section{Contents}

1 Introduction 2

1.1 Market Observations . . . . . . . . . . . . . . . . . . . . . . . . 2

1.2 Objectives and Related Studies . . . . . . . . . . . . . . . . . . . . . 4

2 Black-Scholes Model with an EA Jump 5

2.1 Implied Volatility . . . . . . . . . . . . . . . . . . . . . . . . . . 6

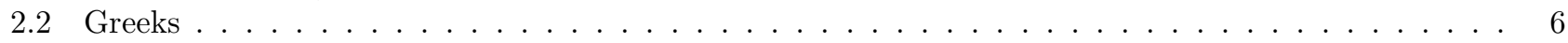

3 Incorporating EA Jumps into Other Models 8

3.1 Extensions of the Kou Model . . . . . . . . . . . . . . . . . . . . . . . . 8

3.2 Pricing via the Characteristic Function . . . . . . . . . . . . . . . . . . . . . . . 10

4 Pre-Earnings Announcement Implied Volatility Properties 11

4.1 Implied Volatility Bounds . . . . . . . . . . . . . . . . . . . . . . . . 11

4.2 Small and Large Strikes Asymptotics . . . . . . . . . . . . . . . . . . . . . . . . 13

*Industrial Engineering \& Operations Research Department, Columbia University, New York, NY 10027, USA. E-mail: leung@ ieor.columbia.edu. Corresponding author.

${ }^{\dagger}$ Industrial Engineering \& Operations Research Department, Columbia University, New York, NY 10027, USA. E-mail: ms4164@columbia.edu. 
5 Calibration and Parameter Estimators 15

5.1 Model Calibration . . . . . . . . . . . . . . . . . . . . . . . 15

5.2 Analytic Estimators under the Extended BS Model . . . . . . . . . . . . . . . . . . . . . 17

5.3 Implied EA jump Distribution and Risk Premia . . . . . . . . . . . . . . . . . . . 18

6 American Options 19

6.1 American Option Price and Exercising Boundary . . . . . . . . . . . . . . . . . 19

6.2 Analytical Approximations . . . . . . . . . . . . . . . . . . . . . . . 20

$\begin{array}{ll}\text { Appendix } & 22\end{array}$

\section{Introduction}

Public companies routinely release summaries of their operations and performance, including income statements, balance sheets, and other reports. Such events are commonly referred to as earnings announcements. In the market session following a scheduled announcement1, empirical studies (see, for example, Patell and Wolfson (1984)) suggest that the opening stock price can move drastically. For instance, Dubinsky and Johannes (2006) study a sample of stocks and report that the variance of stock price returns on the earnings announcement date is over five times greater than those on other dates. In addition to the immediate price impact, earnings releases may also affect the drift of the stock price over a longer horizon. This empirical observation is commonly referred to as the post-earnings-announcement drift; see Chordia and Shivakumar (2006) and references therein.

Since many public companies also have options written on their stock prices, this motivates us to investigate the problem of pricing equity options prior to an earnings announcement (EA). As options are intrinsically forward-looking contracts, their prices should account for the uncertain stock price impact of an upcoming earnings release. A natural question is how to extract some information on such an impact from observed options prices, especially a few days before the announcement. Since traders often quote or study the implied volatility (IV) for each option, in practice it is important to better understand the behavior of the pre-earnings announcement implied volatility (PEAIV).

\subsection{Market Observations}

One main feature of the PEAIV is that it tends to rise, often rather drastically, up until the announcement date. In Figure 1, we show the price and IV of the front month (i.e. with the nearest maturity date) atthe-money (ATM) call option written on IBM. Following an earnings announcement on July 17, 2013, the option expires on the Friday in the same week. As we can see on the right panel, the IV increases rapidly from $30 \%$ to over $70 \%$ as time approaches the earnings announcement, and then drops significantly to close to $20 \%$ after the earnings report is released. At the same time, the ATM call price stays well above zero even though the time to maturity is very short (Figure 1 (left)). Moreover, we observe that the IV reaches a maximum just before an earnings event.

Let us briefly discuss the intuitions behind these observations. First, the market price of an option expiring after earnings announcement reflects the possibility of a jump in the stock price on the announcement date. Since the Black-Scholes formula, which is used to convert option prices to IVs, assumes a log-normal model for the underlying price without jumps, the only way to account for the deterministically timed yet random jump in the stock price is to apply a higher volatility parameter. Furthermore, since the volatility parameter in the Black-Scholes formula is coupled with time-to-maturity, an even higher volatility parameter is needed as time progresses. As a result, even though the stock price may

\footnotetext{
${ }^{1}$ Earnings reports are commonly released after the market closes. Therefore, the price impact of earnings are first reflected at the beginning of the next market session.
} 
not experience a higher (historical) volatility or clear directional movements before earnings, the (riskneutral) IV tends to increase. However, as soon as the earnings announcement is made, the jump in the stock price is realized and the volatility effect will also disappear.

To illustrate that the increasing PEAIV is more than a coincidence, in Figure 2 (left) we plot the time series of the IV of the ATM call option for IBM from January 1996 to August 2013. In the same figure, we mark in red the IV values on days during which earnings announcements were made. As we can see, spikes in volatility most often coincide with the earnings release dates. We can also gain some insight from the shape of the implied volatility surface. In Figure 2 (right), we show the IV surface of IBM on July 15, 2013, two days before an earnings release. It is also clear that the front month options, which have 1 day to expiration, have very high volatility. The implied volatility is significantly lower for longer-term options, suggesting a less pronounced effect of the upcoming earnings announcement.
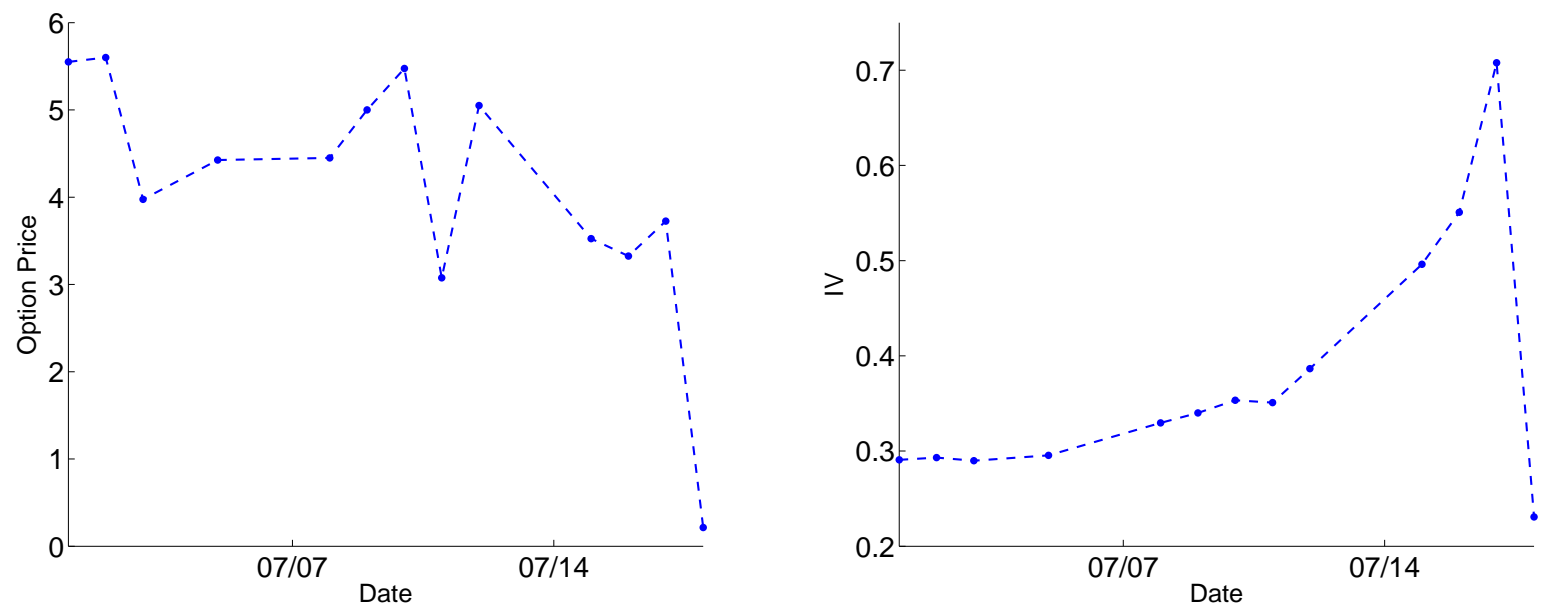

Figure 1: The price (left) and implied volatility (right) of the front month ATM option on IBM with expiration date July 18, 2013. The earnings announcement date is July 17, 2013.
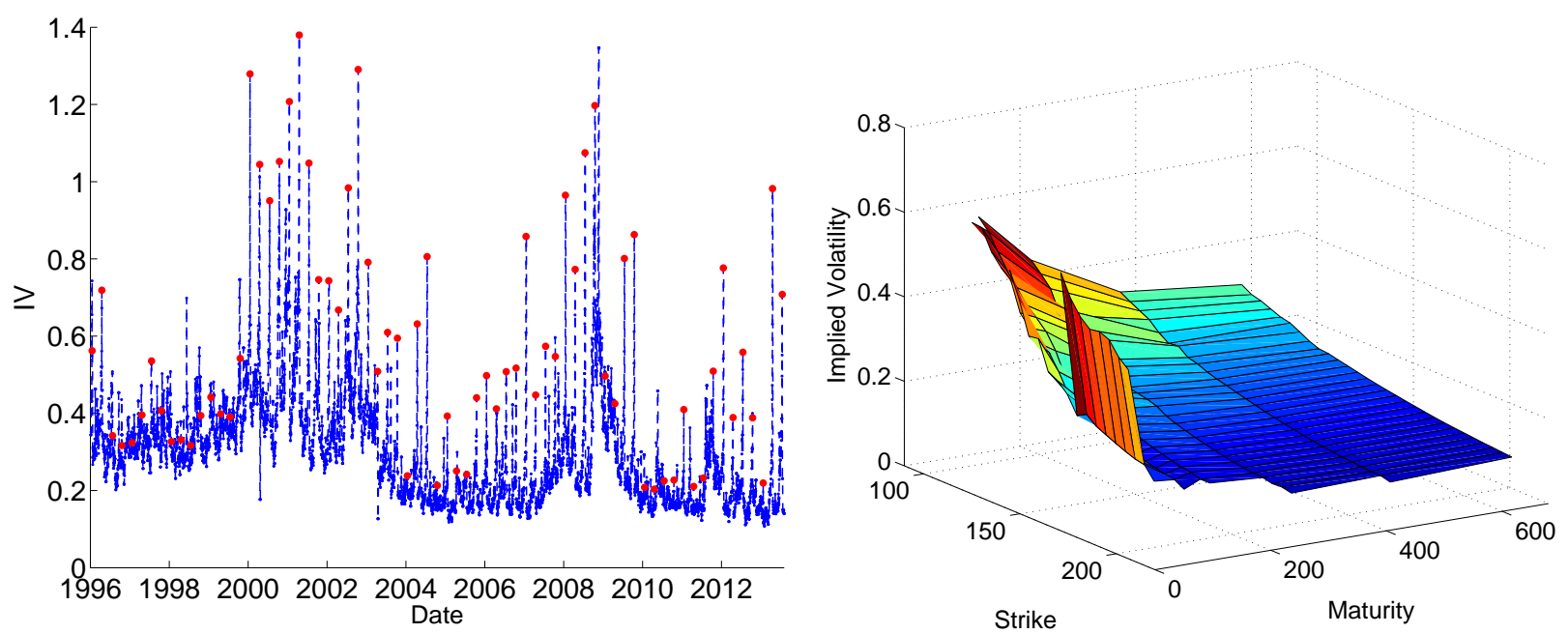

Figure 2: The time series (left) of the IV of the front month ATM call on IBM. The red dots mark the value of the IV on EA dates. The IV surface (right) of IBM at the market close on July 15, 2013, two days before an EA. 


\subsection{Objectives and Related Studies}

These market observations call for a pricing framework that explicitly incorporates the jump to be realized on the earnings announcement date. In this paper, we analyze an option pricing framework that accounts for the price impact of an earnings announcement, with emphasis on the behavior of the implied volatility prior to the event. Specifically, we introduce a random-sized jump scheduled on the earnings announcement date to represent the shock to the company stock price. The introduction of a jump related to earnings is consistent with prior empirical studies (Maheu and McCurdy (2004); Piazzesi (2005); Dubinskv and Johannes (2006); Lee and Mykland (2008)). We first apply this idea to the Black and Scholes (1973) (BS) model, where we obtain formulas for the pre-EA European option price, its IV and various Greeks. In this model, we find that the IV increases with a specific pattern as time approaches the announcement date. We further incorporate additional random-time jumps in the stock price by extending the Kou (2002) double-exponential (DE) jump-diffusion model to incorporate an EA jump. Specifically, we assume that the EA jump size for the log stock price is either a DE or Gaussian random variable. In both cases, analytic option pricing formulas are derived and used to generate IVs.

To better understand the behavior of the PEAIV, we derive analytic bounds and asymptotics for extreme strikes for the IV under a general class of stock price models with an EA jump. In the bounds, we observe the explicit dependence on EA jump parameters and a common time-dependence of the PEAIV across these models. In addition, we calibrate a number of models, namely, the extended Heston and Kou models with an EA jump, to market option prices. Our results demonstrate a more adequate fit of the entire PEAIV surface, as compared to these models without the EA jump. Furthermore, we extract the risk-neutral distribution of the EA jump and compare it to its historical distribution. The discrepancy between the two distributions can be interpreted as a risk premium for the jump risk due to earnings. We conclude the paper analyzing the pricing of American options before and after an EA date. For implementation, we discuss a numerical method, as well as an analytical approximation based on the work of Barone-Adesi and Whaley (1987).

There is a wealth of literature in empirical finance and accounting that examines how earnings announcements affect stock and option prices. Most of the studies focus on the informational content, market reactions and price patterns associated with earnings releases. Systematic reviews of the empiri-

cal studies can be found in Dubinsky and Johannes (2006), Barth et al. (2011), Rogers et al. (2009) and Billings and Jennings (2011). For the price impact of news on futures, we refer to Chatrah et al. (2009) and references therein. Below, we summarize a number of related studies that focus on option prices prior to an earnings announcement.

The model by Patell and Wolfson (1981) (PW) is among the first empirical studies on the prices of options in the presence of an earnings announcement. They build on the pricing models of Black and Scholes (1973) and Merton (1973), and assume the instantaneous volatility as a deterministic piece-wise constant function of time, with two volatility levels over two time windows around the earnings release in order to reflect the uncertainty surrounding the earnings impact. Since their seminal work, other papers have adopted the same model or its variations and performed empirical tests (see Donders and Vorst (1996), Isakov and Perignon (2001), Barth et al. (2011), among others). In particular, Barth et al. (2011) extend PW's model by allowing the deterministic piece-wise constant volatility to take different values before, during, and after the announcement date. In all these empirical studies, there are no jumps incorporated in the stock price at anytime. Moreover, without stochastic volatility or jumps in the stock price, the IV will not exhibit any skew, though this is commonly observed in the options market.

In this paper, we adopt a different approach by incorporating a deterministic-time random jump in the stock price dynamics. We consider different distributions of the scheduled earnings jump as well as different underlying stock price dynamics before and after the EA date. Our study generalizes that of Dubinsky and Johannes (2006), which focuses on the BS model with a Gaussian EA jump and provide a 
formula for the time-deterministic implied volatility function. Our main contributions include analytical option pricing formulas when the underlying stock price admits doubly exponential jumps over time (the Kou model), along with a double-exponential or Gaussian EA jump on the announcement date (see Theorem 3.1). Furthermore, we derive analytic bounds and asymptotics for the pre-EA implied volatility under various models (see Propositions 4.1 and 4.2). In addition, we study the pricing of pre-earnings American options under the Kou model and discuss an analytic approximation to the option price by extending the method of Barone-Adesi and Whaley (1987).

Our analysis draws motivation from empirical studies and market observations to introduce more sophisticated models that help reproduce the entire implied volatility surface accurately and tractably. For calibration, we employ recent options data from the whole observed IV surface, instead of ATM only options used in Dubinsky and Johannes (2006). Using earnings data from 1994 to 2013, we also compare the risk-neutral distribution of the EA jump to its historical distribution. The framework and methods introduced herein can be readily generalized to other option pricing models. They can also be useful for monitoring the PEAIV, and estimating the historical and risk-neutral distributions of the EA jump and the associated risk premium.

The rest of the paper is structured as follows. In Section 2, we extend the Black-Scholes model by incorporating an EA jump in the stock price, and derive the corresponding implied volatility function and Greeks. In Section 3, we discuss the extensions of other models, including the Kou and Heston models. In Section 4, we study the bounds and asymptotics for the IV surface under various models. In Section 5, we discuss our calibration results from the extended BS, Kou, and Heston models, and compare the risk-neutral and historical distributions of EA jumps. In Section 6, we study the valuation of American options prior to an earnings announcement. The Appendix contains the proofs and details for our analytical results.

\section{Black-Scholes Model with an EA Jump}

The effect of a scheduled earnings announcement on the stock price is modeled by a deterministic-time random-size price jump in the price dynamics. Naturally, this modification can be applied to virtually any model, and we start with an extension of the Black and Scholes (1973) model.

Let $\left(W_{t}\right)_{t \geq 0}$ be a standard Brownian motion defined on the risk-neutral probability space $(\Omega, \mathcal{F}, \mathbb{Q})$. Throughout, we assume a constant risk-free rate $r \geq 0$. Let $T_{e}$ be the EA date, and we call the r.v. $Z_{e}$ the EA jump, which is the jump size of the log stock price immediately after the earnings announcement. We assume $Z_{e}$ to be independent of $W$. Under the risk-neutral measure, the company stock price $\left(S_{t}\right)_{t \geq 0}$ evolves according to

$$
\frac{d S_{t}}{S_{t_{-}}}=r d t+\sigma d W_{t}+d\left(1_{\left\{t \geq T_{e}\right\}}\left(e^{Z_{e}}-1\right)\right),
$$

where $1_{\{\cdot\}}$ denotes the indicator function, $r$ is the positive constant interest rate, and $\sigma$ represents the constant volatility. The martingale condition $S_{0}=\mathbb{E}\left\{e^{-r t} S_{t}\right\}, 0 \leq t \leq T$, where $\mathbb{E}\{\cdot\}$ denotes the expectation under $\mathbb{Q}$, implies that $\mathbb{E}\left\{e^{Z_{e}}\right\}=1$.

In this section, we assume that $Z_{e}$ is normally distributed. This yields closed form expressions for

the price and the IV surface. The martingale condition $\mathbb{E}\left\{e^{Z_{e}}\right\}=1$ implies that $Z_{e} \sim N\left(-\frac{\sigma_{e}^{2}}{2}, \sigma_{e}^{2}\right)$, so the EA jump is parametrized by $\sigma_{e}$ only. We notice that, for $T \geq T_{e}$,

$$
\log \left(\frac{S_{T}}{S_{t}}\right) \sim N\left(\left(r-\frac{\sigma^{2}}{2}-\frac{\sigma_{e}^{2}}{2(T-t)}\right)(T-t), \sigma^{2}(T-t)+\sigma_{e}^{2}\right) .
$$


Therefore, the price of a European call with strike $K$ and maturity $T$ is given by

$$
C\left(t, S_{t}\right)=C_{B S}\left(T-t, S_{t} ; \sqrt{\sigma^{2}+\frac{\sigma_{e}^{2}}{T-t}}, K, r\right), \quad 0 \leq t<T_{e}
$$

where $C_{B S}(\tau, S ; \sigma, K, r)$ represents the usual BS formula with time to maturity $\tau$ and spot price $S$.

\section{$2.1 \quad$ Implied Volatility}

The price formula (2.3) allows us to express the implied volatility (IV) as the deterministic function

$$
I(t ; K, T)= \begin{cases}\sqrt{\sigma^{2}+\frac{\sigma_{e}^{2}}{T-t}} & 0 \leq t<T_{e} \\ \sigma & T_{e} \leq t<T .\end{cases}
$$

As we can see, although the IV surface, for a fixed time $t$, is flat across strikes, it has a decreasing term structure in $T$. In addition, the IV for any particular option increases in time as we approach the earnings announcement. Alternatively, the option price formula (2.3) can be obtained as if the stock has no jump and follows the dynamics

$$
\frac{d S_{t}}{S_{t}}=r d t+I(t ; K, T) d W_{t}
$$

This model is used in Patell and Wolfson (1981), who assume $I(t ; K, T)$ to be a deterministic, piece-wise constant function to reflect the impact of earnings on the implied volatility.

In Figure 3 (left), we compare the IV function in (2.4) for fixed $(K, T)$, against the IV time series of the front month ATM IBM option 2 with expiration date $7 / 18 / 2013$. The parameters of the model have been chosen by a least-square regression. As we can see, the observed ATM IV and the model IV increase in a similar fashion. In the same figure (right), we also compare the IV function in (2.4) for fixed $(t, K)$, against the term structure of the IV of the ATM IBM call option on 7/17/2013 - just before the earnings announcement and one day before the expiration date. Again, the parameters of the model are obtained via an additional least-square regression and they differ from the one obtained from the IV time series analysis. The model term structure approximates the observed one even if the IV surface presents no skew and a term structure which allows only for the functional form (2.4).

\subsection{Greeks}

In order to understand the sensitivity of options prices approaching the earnings announcement, we derive and analyze the Greeks based on the price function (2.3).

For a call or put with maturity $T$ after the EA date $T_{e}$, the Delta and Gamma are the simply the Black-Scholes Delta and Gamma functions, but with the volatility parameter set at higher value $I(t)$, for $t<T_{e}$. On the other hand, we notice that there are two parameters related to the volatility of the stock price. Consequently, in addition to the standard Black-Scholes Vega, we introduce the EA-Vega

$$
\mathcal{V}_{e} \equiv \frac{\partial C}{\partial \sigma_{e}}=\frac{1}{\sqrt{T-t} \sqrt{\frac{\sigma^{2}(T-t)}{\sigma_{e}^{2}}+1}} \mathcal{V}_{B S}(T-t, S ; I(t), K, r),
$$

where $\mathcal{V}_{B S}(\tau, S ; \sigma, K, r)=S \phi\left(d_{1}\right) \sqrt{\tau}$ represents the usual Black-Scholes Vega function with spot price $S$ and time-to-maturity $\tau$.

\footnotetext{
${ }^{2}$ The IVs of the ATM call and put are observed to be very close but not identical. Each point of the time series represents the average of the two IVs.
} 

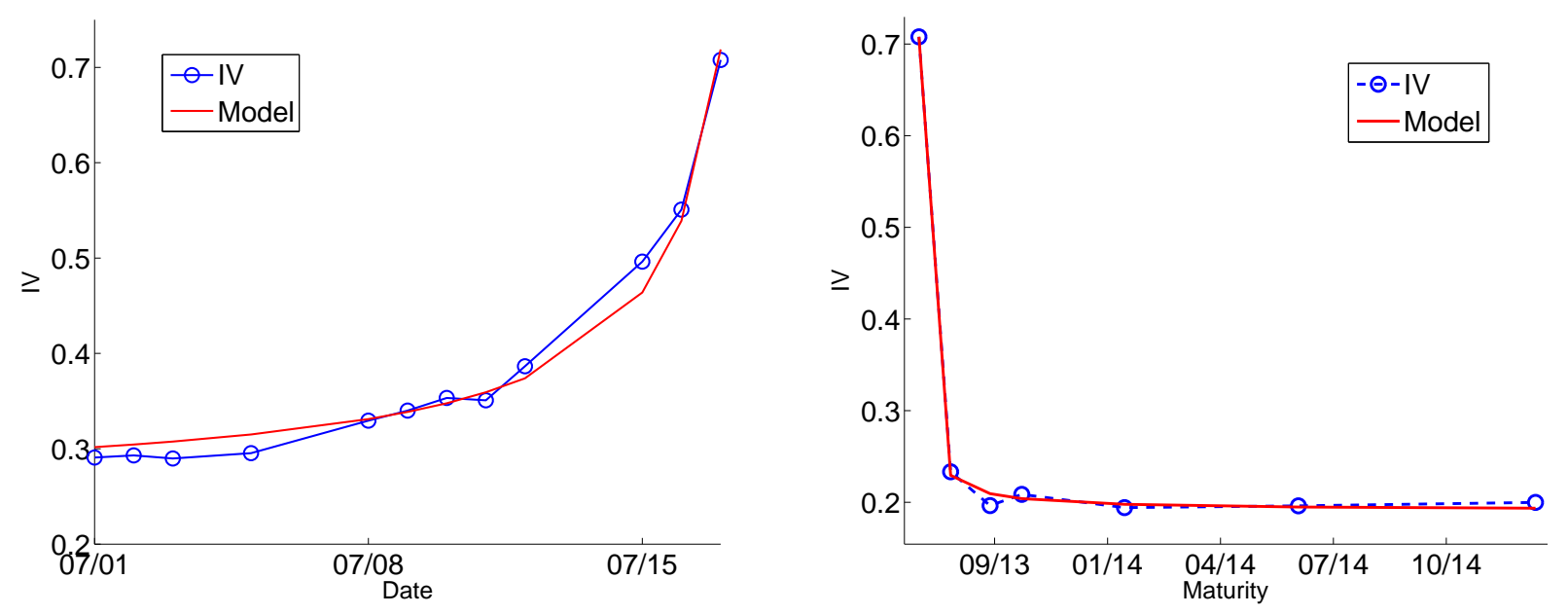

Figure 3: Left: The IV function (2.4) (solid line) prior to an EA, with $\sigma=0.2538, \sigma_{e}=0.0424$ and expiration date $7 / 18 / 2013$, as compared to the market-observed IV (circle) of the front month IBM ATM option with the same expiration date. Right: The term structure of the IV (solid) according to (2.4), with $\sigma=0.1912, \sigma_{e}=0.0429$, on $7 / 17 / 2013$, compared to the market-observed term structure (circle) for ATM IBM options on the same date.

For the Theta of a call, we obtain

$$
\Theta \equiv \frac{\partial C}{\partial t}=\Theta_{B S}(T-t, S ; I(t), K, r)+\frac{1}{2 I(t)}\left(\frac{\sigma_{e}}{T-t}\right)^{2} \mathcal{V}_{B S}(T-t, S ; I(t), K, r), \quad 0 \leq t<T .
$$

where $\Theta_{B S}(\tau, S ; \sigma, K, r)=-S \frac{\sigma}{2 \sqrt{\tau}} \phi\left(d_{1}\right)-r K e^{-r \tau} \Phi\left(d_{2}\right)$ is the Black-Scholes Theta function. First, we note that $\Theta_{B S}(T-t, S ; I(t), K, r) \leq \Theta \leq 0$. The left part of the inequality is a direct consequence of (2.5) while the right part is a consequence of the fact that the option price is decreasing in time. On the other hand, it is not true in general that $\Theta \leq \Theta_{B S}(S, K, r, T-t, \sigma)$. This means that the option may lose value less rapidly over time than an option priced with a lower volatility. To illustrate this point, we suppose $r=0$, and the Black-Scholes PDE implies that

$$
\frac{\partial C}{\partial t}=-\sigma^{2} S^{2} \frac{\partial^{2} C}{\partial S^{2}}=-\sigma^{2} S^{2} \Gamma_{B S}(T-t, S ; I(t), K, 0) .
$$

On the other hand, we also have $\Theta_{B S}(T-t, S ; \sigma, K, 0)=-\sigma^{2} S^{2} \Gamma_{B S}(T-t, S ; \sigma, K, 0)$. Therefore,

$$
\frac{\partial C}{\partial t}-\Theta_{B S}(T-t, S ; \sigma, K, 0)=-\sigma^{2} S^{2}\left(\Gamma_{B S}(T-t, S ; I(t), K, 0)-\Gamma_{B S}(T-t, S ; \sigma, K, 0)\right) .
$$

For ATM options, we have $\Gamma_{B S}(T-t, S ; \sigma, S, 0)=\frac{1}{\sqrt{2 \pi} \sigma S} e^{-\frac{\sigma^{2}(T-t)}{2}}$, which is decreasing in $\sigma$. This implies that $\frac{\partial C}{\partial t}>\Theta_{B S}(T-t, S ; \sigma, S, 0)$ for $\sigma_{e}>0$, implying a less rapid time decay in the option value. In fact, the same is true for other puts and calls whose $\Gamma_{B S}$ is decreasing in $\sigma$.

In Figure 4 (left), we illustrate $\Theta$ as a function of the spot price for a call with 5 days to maturity. For comparison, we plot two additional benchmarks based on the BS Theta with different volatility parameter values $I(0)$ and $\sigma$, respectively. As expected from (2.5), the time-decay of the call price is less severe than $\Theta_{B S}(T, S ; I(0), K, r)$. In addition, for spot prices near the strike $K$, we observe that $\Theta>\Theta_{B S}(T, S ; \sigma, S, r)>\Theta_{B S}(T, S ; I(0), S, r)$. Figure凹(right) shows how $\Theta$ changes in time for an ATM ( $S=K=100)$ call, up to one day before earnings. Again, we notice the same dominance of Theta's, 
but their differences increase as time approaches the EA date. Interestingly, $\Theta$, which accounts for the EA jump, appears to be significantly more constant as compared to the other two BS Theta's.
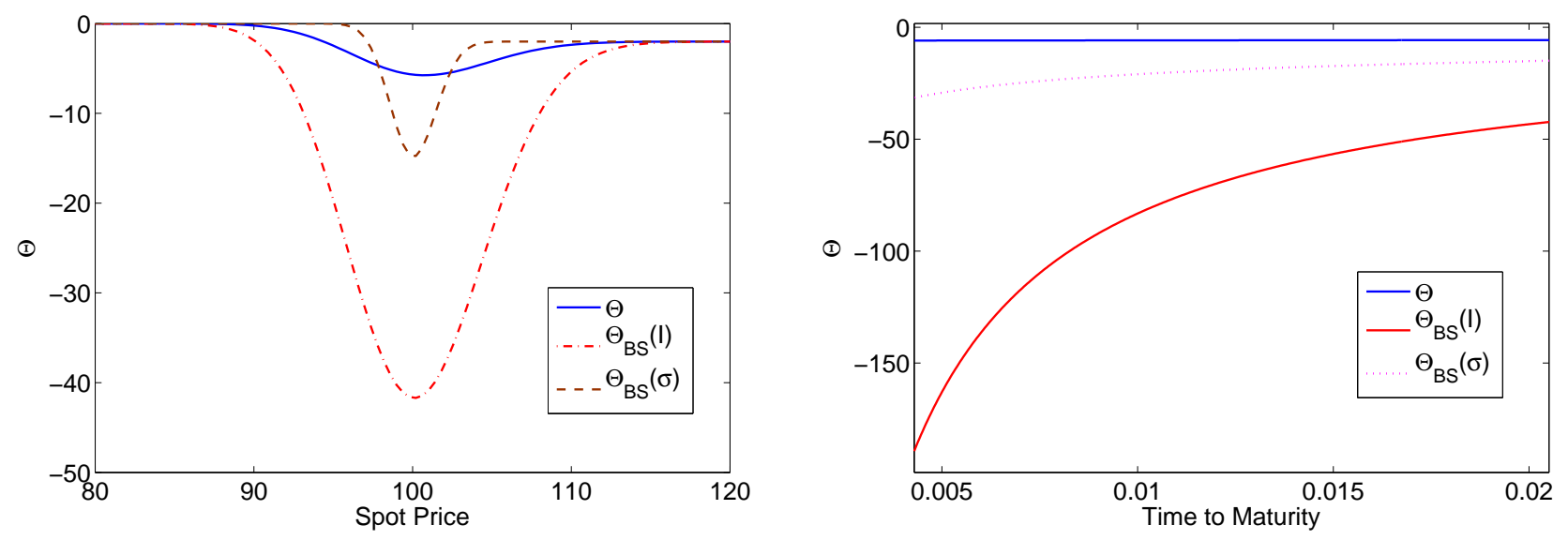

Figure 4: The shape of $\Theta$ and $\Theta_{B S}$ changes w.r.t. the spot price (with $T=\frac{5}{252}$ ) and time to maturity (with $S=K=100)$. In both panels, we see that $\Theta$ for ATM options is less negative than the ordinary BS Theta $\Theta_{B S}$. Common parameters: $r=0.02, \sigma=0.1, \sigma_{e}=0.04$.

\section{Incorporating EA Jumps into Other Models}

Although the extended BS model (2.1) is capable of showing an increasing IV approaching an earnings announcement, the IV has no skew and its term structure only admits the particular two-parameter functional form $\sqrt{\sigma^{2}+\frac{\sigma_{e}^{2}}{T-t}}$. On the other hand, in addition to the scheduled jump, the stock price may also experience randomly timed jumps which cannot be adequately captured by diffusion. This has motivated many models that incorporate jumps of various distributions into the stock price dynamics, with notable examples in the Merton (1976), Kou (2002), Variance Gamma (Madan et al. (1998)), CGMY (Carr et al. (2002))) models. In this section, we present an analytic formula for pricing European options under an extension of the jump-diffusion model of Kou (2002), and also discuss the pricing of options prior to an earnings announcement using a transform method.

\subsection{Extensions of the Kou Model}

We consider an extension of the Kou model and derive an analytic formula for the price of a European option with an EA jump. Under this model, the risk-neutral terminal stock price follows

$$
\log \left(\frac{S_{T}}{S_{0}}\right)=-\frac{\sigma^{2}}{2} T+\sigma W_{T}-m \kappa T+\sum_{i=1}^{N_{T}} J_{i}+1_{\left\{t \geq T_{e}\right\}}\left(Z_{e}-\log \left(\mathbb{E}\left\{e^{Z_{e}}\right\}\right)\right),
$$

where each jump $J_{i} \sim D E\left(p, \lambda_{1}, \lambda_{2}\right)$ is double-exponentially distributed with the p.d.f. $f_{J_{i}}(x)=$ $1_{\{x \geq 0\}} p \lambda_{1} e^{-\lambda_{1} x}+1_{\{x \leq 0\}}(1-p) \lambda_{2} e^{\lambda_{2} x}$. The number of randomly timed jumps is modeled by $N_{T} \sim$ $\operatorname{Poi}(\kappa T)$. To ensure that the martingale condition holds, we set $m=p \frac{\lambda_{1}}{\lambda_{1}-1}+(1-p) \frac{\lambda_{2}}{\lambda_{2}+1}-1$. We shall consider separately two distributions for the EA log jump size $Z_{e}$ : (i) double-exponential $Z_{e} \sim D E\left(u, \eta_{1}, \eta_{2}\right)$, and (ii) Gaussian $Z_{e} \sim N\left(0, \sigma_{e}^{2}\right)$. 
Let $C(S)=\mathbb{E}\left\{e^{-r T}\left(S_{T}-K\right)^{+} \mid S_{0}=S\right\}$ be the price of the European call option at time $t=0$ when the spot price is $S$. We now present the price formula when the EA jump $Z_{e}$ is a double exponential random variable.

Proposition 3.1. Suppose the terminal stock price follows (3.1), with $Z_{e} \sim D E\left(u, \eta_{1}, \eta_{2}\right)$ and $\eta_{1} \neq$ $\lambda_{1}, \eta_{2} \neq \lambda_{2}$. Then, the European call option price is given by

$$
\begin{aligned}
C(S)= & e^{-\alpha} S \Upsilon\left(S, K, r+\sigma^{2}, T, \sigma, \hat{\kappa}, \hat{p}, \hat{\lambda}_{1}, \hat{\lambda}_{2}, \hat{u}, \hat{w}, \hat{\eta}_{1}, \hat{\eta}_{2}\right) \\
& -K e^{-r T} \Upsilon\left(S, K, r, T, \sigma, \kappa, p, \lambda_{1}, \lambda_{2}, u, w, \eta_{1}, \eta_{2}\right),
\end{aligned}
$$

where the function $\Upsilon$ is given in Appendix A.1, and the constants are

$$
\begin{gathered}
\hat{\eta}_{1}=\eta_{1}-1, \quad \hat{\eta}_{2}=\eta_{2}+1, \quad \hat{\lambda}_{1}=\lambda_{1}-1, \quad \hat{\lambda}_{2}=\lambda_{2}+1, \quad \hat{u}=u \frac{\eta_{1}}{\eta_{1}-1}, \quad w=1-u, \quad \hat{w}=w \frac{\eta_{2}}{\eta_{2}+1}, \\
\hat{\kappa}=(m+1) \kappa, \quad \hat{p}=\frac{\lambda_{1}}{\lambda_{1}-1} \frac{p}{m+1}, \quad \alpha=\log \left(u \frac{\eta_{1}}{\eta_{1}-1}+w \frac{\eta_{2}}{\eta_{2}+1}\right) .
\end{gathered}
$$

When the jump size parameters of the EA and randomly timed jumps are the same, i.e. $\eta_{1}=\lambda_{1}$ or $\eta_{2}=\lambda_{2}$, an analytic pricing formula can also be derived but omitted here. In practice, the parameters $\lambda_{1}$, $\lambda_{2}$ are usually of an order of magnitude greater than $\eta_{1}$ and $\eta_{2}$, as we will observe from our calibration in Section 5 .

Alternatively, if the EA jump is normally distributed, i.e. $Z_{e} \sim N\left(0, \sigma_{e}^{2}\right)$, then one can directly adapt the result from Kou (2002) to account for the EA jump. Specifically, the EA jump parameter $\sigma_{e}$ can be incorporated in the volatility coefficient of the Brownian motion $W_{T}$ :

$$
\log \left(\frac{S_{T}}{S_{0}}\right) \stackrel{d}{=}\left(-\frac{\sigma^{2}+\frac{\sigma_{e}^{2}}{T}}{2}-m \kappa\right) T+\sqrt{\sigma^{2}+\frac{\sigma_{e}^{2}}{T}} W_{T}+\sum_{i=1}^{N_{T}} J_{i} .
$$

From this, we see that the resulting analytic formula is in fact identical to the original formula without the EA jump, except with $\sigma$ replaced by $\sqrt{\sigma^{2}+\frac{\sigma_{e}^{2}}{T}}$.

The analytic formula (3.2) allows for the fast computation of the price, and simultaneously its delta, $\Delta=e^{-\alpha} \Upsilon(\cdot)$, where $\Upsilon(\cdot)$ is the first term on RHS of (3.2). In Table 3.1, we apply (3.2) to calculate option prices and we report the corresponding implied volatilities. As we can see, the IV decreases as time to maturity increases, which is typical of the IV before an earnings announcement. In addition, the IV skew becomes flatter as maturity increases. In Figure 5, we plot the IV of the ATM option when the underlying follows (3.1) with either a Gaussian or DE EA jump. For comparison, we have chosen the jumps parameters so that the variances of the Gaussian and DE EA jumps coincide. We notice that the IVs have similar dependence on time. Comparing Figures 3 and 5, it is natural to wonder whether the IV increases in time in a similar fashion, not only in the extended Black-Scholes and Kou, but also in other models. This motivates us to explore the properties of the IV under different models in Section 4 . 


\begin{tabular}{r|rrrrrrrr}
\hline$K \backslash T$ & \multicolumn{2}{|c}{1 Week } & \multicolumn{2}{c}{ 1 Month } & \multicolumn{2}{c}{ 3 Months } & \multicolumn{2}{c}{ 1 Year } \\
\hline & \multicolumn{1}{|c}{$C$} & \multicolumn{1}{c}{$I V$} & \multicolumn{1}{c}{$C$} & \multicolumn{1}{c}{$I V$} & \multicolumn{1}{c}{$C$} & \multicolumn{1}{c}{$I V$} & \multicolumn{1}{c}{$C$} & \multicolumn{1}{c}{$I V$} \\
\hline 90 & 11.031 & 0.799 & 11.380 & 0.425 & 12.348 & 0.300 & 16.050 & 0.239 \\
92.5 & 8.958 & 0.767 & 9.400 & 0.415 & 10.529 & 0.297 & 14.485 & 0.239 \\
95 & 7.048 & 0.738 & 7.598 & 0.407 & 8.871 & 0.295 & 13.027 & 0.239 \\
97.5 & 5.357 & 0.714 & 6.007 & 0.401 & 7.384 & 0.294 & 11.675 & 0.239 \\
100 & 3.945 & 0.699 & 4.651 & 0.397 & 6.075 & 0.293 & 10.428 & 0.239 \\
102.5 & 2.849 & 0.696 & 3.536 & 0.396 & 4.942 & 0.292 & 9.284 & 0.239 \\
105 & 2.047 & 0.704 & 2.651 & 0.396 & 3.979 & 0.292 & 8.240 & 0.238 \\
107.5 & 1.475 & 0.719 & 1.968 & 0.399 & 3.173 & 0.292 & 7.292 & 0.238 \\
110 & 1.069 & 0.738 & 1.455 & 0.403 & 2.509 & 0.293 & 6.434 & 0.238 \\
\hline
\end{tabular}

Table 1: Option prices and IVs under model (3.1). Prices are computed via formula (3.2). Parameters: $r=2 \%$, $S_{0}=100, \kappa=10, p=0.6, \lambda_{1}=60, \lambda_{2}=50, u=0.55, \eta_{1}=15, \eta_{2}=12, \sigma=20 \%$.

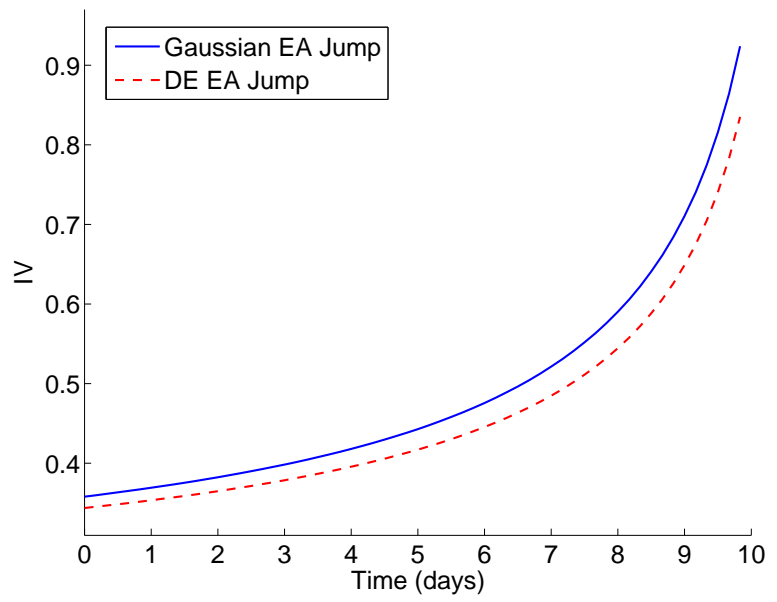

Figure 5: The time-series of the ATM option IV under the extended Kou model (3.1) when the EA and expiration dates are on the 10 th and 11 th days respectively. Parameters: $T_{e}=14$ days, $T=15$ days, $r=2 \%, S_{0}=100$, $\kappa=10, p=0.5, \lambda_{1}=50, \lambda_{2}=50, u=0.5, \eta_{1}=25, \eta_{2}=25, \sigma=20 \%$.

\subsection{Pricing via the Characteristic Function}

In general, let us write the terminal stock price as $S_{T}=S_{0} e^{X_{T}+Z_{e}}$. If $X_{T}$ and $Z_{e}$ are independent, and they both admit analytic characteristic functions, then we obtain the characteristic formula of the $\log$-price

$$
\Psi(\omega):=\mathbb{E}\left\{e^{i \omega \log \left(\frac{S_{T}}{S_{0}}\right)}\right\}=e^{\hat{\psi}(\omega)+\psi_{e}(\omega)},
$$

where

$$
\hat{\psi}(\omega):=\log \left(\mathbb{E}\left\{e^{\mathrm{i} \omega \log \left(X_{T}\right)}\right\}\right), \quad \text { and } \quad \psi_{e}(\omega):=\log \left(\mathbb{E}\left\{e^{\mathrm{i} \omega \log \left(Z_{e}\right)}\right\}\right) .
$$

It is then possible to make use of available methods to price vanilla as well as exotic options. For example, the methods of Carr and Madan (1999), Duffie and Singleton (2000), Lee (2004), and Raible (2000), among others, can be used to price European options. Alternatively, the methods developed in Jackson et al. (2008) or Lord et al. (2008) can be adapted to price both European and American options in models incorporating the jump $Z_{e}$. 
In the following sections, we will also consider an extension of the Heston model with dynamics

$$
\begin{aligned}
\frac{d S_{t}}{S_{t}} & =r d t+\sigma_{t} d W_{t}+d\left(1_{\left\{t \geq T_{e}\right\}}\left(e^{Z_{e}}-1\right)\right), \\
d \sigma_{t}^{2} & =\nu\left(\vartheta-\sigma_{t}^{2}\right) d t+\zeta \sigma_{t} d \tilde{W}_{t},
\end{aligned}
$$

where $W$ and $\tilde{W}$ are standard Brownian motions, with $\mathbb{E}\left\{d W_{t} d \tilde{W}_{t}\right\}=\rho d t$, and $Z_{e}$ is independent of both $W$ and $\tilde{W}$. Write the option price as

$$
\begin{aligned}
C(0, S) & =\int_{-\infty}^{\infty}\left(e^{\log (S+x)}-K\right)^{+} f(x) d x \\
& =\left(e^{\cdot}-K\right)^{+} * f(-\cdot)(\log (S))
\end{aligned}
$$

where $f$ denotes the p.d.f. of $\log \left(\frac{S_{T}}{S_{0}}\right)$ and $*$ denotes the convolution operator. Denoting with $\mathcal{F}(g(x)) \equiv$ $\int_{\mathbb{R}} e^{-i \omega x} g(x) d x$ the Fourier operator acting on the function $g$, we can then write the price of the European call as

$$
C(0, S)=e^{\gamma \log (S)} \mathcal{F}^{-1}\left\{\mathcal{F}\left\{e^{-\gamma x}\left(S e^{x}-K\right)^{+}\right\} \Psi(\omega-i \gamma)\right\} .
$$

where the introduction of the dampening factor $e^{-\gamma x}$ is necessary because the payoff is not integrable. The same observations have been made in Lord et al. (2008), where (3.4) is implemented as part of a new pricing algorithm. Jackson et al. (2008) also derive the same formula by analyzing the associated pricing PIDE in Fourier space. One can then apply a Fast Fourier transform (FFT) algorithm to price according to (3.4). In addition, these methods can be adapted to price American options, as we will do in Section 6.

\section{Pre-Earnings Announcement Implied Volatility Properties}

The market observations in Figures 1-3 motivate us to analyze some characteristics of the pre-earnings announcement implied volatility. In Section 4.1, we provide upper and lower bounds for the IV under a class of models. In Section 4.2, we study some IV asymptotics with focus on small and large strikes.

\subsection{Implied Volatility Bounds}

For our analysis of the IV bounds of European options, we consider a general framework where the terminal stock price is written in the form

$$
S_{T}=S_{t} e^{X_{t, T}+Z_{e}}
$$

with $S_{t}$ the stock price at time $t<T_{e}$. The martingale condition on $S$ implies that $\left(X_{t, T}\right)_{0 \leq t \leq T}$ satisfies $\mathbb{E}\left\{e^{X_{t, T}}\right\}=e^{r(T-t)}$. The r.v. $Z_{e}$ is a continuous mixture of Gaussian r.v.'s with p.d.f. $f_{Z_{e}}(y)=$ $\int_{\mathbb{R}^{+}} \phi\left(y ;-\frac{\hat{\sigma}^{2}}{2}, \hat{\sigma}\right) \mathbb{G}(d \hat{\sigma})$, where $\phi(\cdot ; a, b)$ represents the p.d.f. of a Gaussian r.v. with mean $a$ and variance $b^{2}$, and $\mathbb{G}(\cdot)$ is a measure over the space $\mathbb{R}^{+}$with $\mathbb{G}[0, \infty)=1$.

Note that we have not specified the distribution of $X_{t, T}$, therefore the base model can be very general. We have the following lower bound for the IV volatility function.

Proposition 4.1. Suppose the terminal stock price $S_{T}$ follows (4.1). Then, the implied volatility $I(t ; K, T)$ admits the lower bound

$$
I(t ; K, T) \geq \frac{\hat{\sigma}_{\text {min }}}{\sqrt{T-t}}, \quad t<T_{e}
$$


where $\hat{\sigma}_{\text {min }}:=\inf \left\{\hat{\sigma} \in \mathbb{R}_{+}: \mathbb{G}[0, \hat{\sigma}]>0\right\}$. In addition, if $X_{t, T}$ is also distributed as a Gaussian mixture, $f_{X_{t, T}}(y)=\int_{\mathbb{R}^{+}} \phi\left(y ; r(T-t)-\frac{\tilde{\sigma}^{2}}{2}, \tilde{\sigma}\right) \mathbb{H}(d \tilde{\sigma})$, then the lower bound improves to

$$
I(t ; K, T) \geq \sqrt{\frac{\tilde{\sigma}_{\min }^{2}+\hat{\sigma}_{\min }^{2}}{T-t}}, t<T_{e},
$$

where $\tilde{\sigma}_{\text {min }}:=\inf \left\{\tilde{\sigma} \in \mathbb{R}_{+}: \mathbb{H}[0, \tilde{\sigma}]>0\right\}$.

We note that $\hat{\sigma}_{\min } \geq 0$, so the bound (4.2) is nontrivial only if $\hat{\sigma}_{\min }>0$. This means that the measure $G$ has zero weight on Gaussian r.v.'s with variance smaller than $\hat{\sigma}_{\min }>0$. Such a condition is satisfied, for example, by any finite mixture of Gaussian r.v.

In addition, we obtain an upper bound for the implied volatility.

Proposition 4.2. Suppose the terminal stock price $S_{T}$ follows 4.1) and assume that both $X_{T}$ and $Z_{e}$ are distributed as continuous Gaussian mixtures,

$$
f_{Z_{e}}(y)=\int_{\mathbb{R}^{+}} \phi\left(y ;-\frac{\hat{\sigma}^{2}}{2}, \hat{\sigma}\right) \mathbb{G}(d \hat{\sigma}), \quad f_{X_{t, T}}(y)=\int_{\mathbb{R}^{+}} \phi\left(y ; r(T-t)-\frac{\tilde{\sigma}^{2}}{2}, \tilde{\sigma}\right) \mathbb{H}(d \tilde{\sigma}) .
$$

Then, the following upper bound for the implied volatility of a European ATM-forward call option, $K=$ $e^{r(T-t)} S_{t}$, holds:

$$
I\left(t ; e^{r(T-t)} S_{t}, T\right) \leq \sqrt{\int_{\mathbb{R}^{+}} \frac{\tilde{\sigma}^{2}}{T-t} \mathbb{H}(d \tilde{\sigma})+\int_{\mathbb{R}^{+}} \frac{\hat{\sigma}^{2}}{T-t} \mathbb{G}(d \hat{\sigma})}, t<T_{e} .
$$

Notable examples of models that satisfy conditions (4.3) include the extended Merton and Heston (when $\rho=0$ ) models. However, we remark that Gaussian mixtures can also be used to approximate other distributions. Moreover, our bounds can serve as analytical benchmarks for the IV under different models. As an example, we derive the explicit expressions for the bounds (4.4) under the Heston model.

According to Propositions 4.1 and 4.2, the IV bounds under different models exhibit similar behaviors as time approaches the earnings announcement. Comparing the bounds to the IV function $I$ in (2.4), it is not too surprising that the simple extended BS model was able to fit the observed ATM IV over time (see Figure 3).

Example 4.3. Assume that $S$ follows the Heston dynamics (3.3). In the case $\rho=0$ it is known that, conditioned on the path of $\left(\sigma_{u}\right)_{t \leq u \leq T}, \int_{t}^{T} \sigma_{u} d W_{u} \sim N\left(0, \tilde{\sigma}^{2}\right)$, where $\tilde{\sigma}^{2} \equiv \int_{t}^{T} \sigma_{u}^{2} d u$. From this we observe that $X_{t, T} \equiv\left(r-\frac{\tilde{\sigma}^{2}}{2(T-t)}\right)(T-t)+\int_{t}^{T} \sigma_{u} d W_{u}$ satisfies the second part of (14.3). In turn, direct computation yields that $\int_{\mathbb{R}^{+}} \frac{\tilde{\sigma}^{2}}{T-t} \mathbb{H}(d \tilde{\sigma})=\vartheta+\frac{\sigma_{t}^{2}-\vartheta}{\nu(T-t)}\left(1-e^{-\nu(T-t)}\right)$. Therefore, for example, in the case of a Gaussian EA jump, the term $\int_{\mathbb{R}^{+}} \frac{\hat{\sigma}^{2}}{T-t} \mathbb{G}(d \hat{\sigma})$ is equal to $\frac{\sigma_{e}^{2}}{T-t}$ and the bound 4.4) reads

$$
I\left(t ; e^{r(T-t)} S_{t}, T\right) \leq \sqrt{\vartheta+\frac{\sigma_{t}^{2}-\vartheta}{\nu(T-t)}\left(1-e^{-\nu(T-t)}\right)+\frac{\sigma_{e}^{2}}{T-t}} .
$$

If we instead assume that the jump is distributed as a symmetric double-exponential, $Z_{e} \sim D E\left(\frac{1}{2}, \eta, \eta\right)$, then we use the fact that $Z_{e} \stackrel{d}{=} \sqrt{\frac{2}{\eta^{2}}} \epsilon Z$, where $\epsilon \sim \operatorname{Exp}(1)$ and $Z \sim N(0,1)$ are independent. In fact, in that case, $\mathbb{G}(x)=\eta^{2} x e^{-\frac{\eta^{2} x^{2}}{2}}, \int_{\mathbb{R}^{+}} \frac{\hat{\sigma}^{2}}{T-t} \mathbb{G}(d \hat{\sigma})=\frac{2}{\eta^{2}(T-t)}$, and the bound 4.4) reads

$$
I\left(t ; e^{r(T-t)} S_{t}, T\right) \leq \sqrt{\vartheta+\frac{\sigma_{t}^{2}-\vartheta}{\nu(T-t)}\left(1-e^{-\nu(T-t)}\right)+\frac{2}{\eta^{2}(T-t)}} .
$$


In Figure 6 we plot the explicit bounds (4.5) and (4.6) from Example 4.3. As we can see, the upper bound is relatively close to the model IV curve and shares a very similar time dependence. In particular, when the EA jump is Gaussian (Figure 6, left), the bound is almost indistinguishable from the model IV. To see this, as $t \rightarrow T$, the lower and upper bounds ((4.2) and (4.5) ) share a common leading term $\frac{\sigma_{e}^{2}}{T-t}$. In practical cases as in this example, the term $\int_{\mathbb{R}^{+}} \frac{\tilde{\sigma}^{2}}{T-t} \mathbb{H}(d \tilde{\sigma}) \approx \sigma_{t}^{2}$ is typically at least one order of magnitude smaller than $\sigma_{e}^{2} /(T-t)$ since $T-t$ is very small and $\sigma_{e}$ and $\sigma_{t}$ are of the same order. Also, in Figure 6, we observe that the model IV with a non-zero $\rho$ still admits a similar time behavior and are very close to the bounds (4.5) and (4.6) for the case $\rho=0$. As a curious note, the coefficient $\frac{2}{\eta^{2}}$ of the leading term in (4.6) as $t \rightarrow T$, is exactly the variance of $Z_{e}$ when $Z_{e} \sim D E\left(\frac{1}{2}, \eta, \eta\right)$, the same way that the coefficient $\sigma_{e}^{2}$ of the leading term in (4.5) is the variance of the Gaussian $Z_{e}$. Therefore, an interesting question is whether the rate of change of the IV is approximately proportional to the standard deviation of the EA jump, at least as time approaches the EA date (see also Figure 5).

To conclude, we recall that Propositions 4.1 and 4.2 also give us information about the term structure of the IV. Indeed, for models whose dynamics are time-homogeneous, we observe the relationship: $\frac{\partial I(t ; K, T)}{\partial t}=-\frac{\partial I(t ; K, T)}{\partial T}$. This implies that one should expect a decreasing term structure for options prior to the EA date.
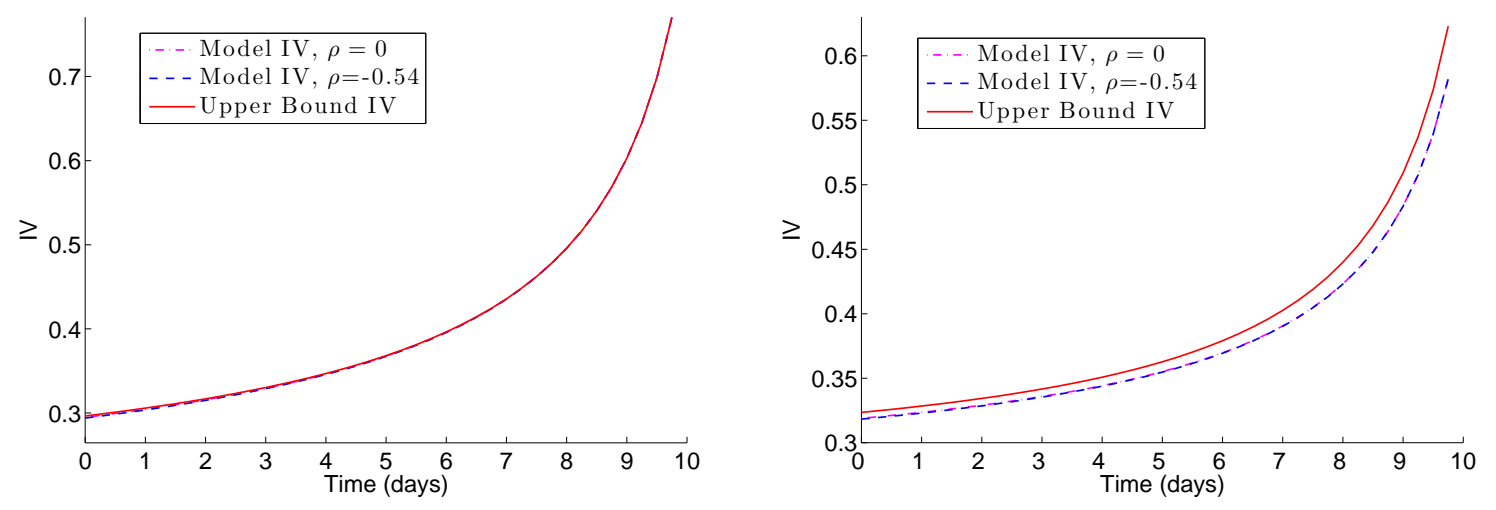

Figure 6: The model IV plotted against the upper bounds in (4.5) and (4.6) under the extended Heston model with a Gaussian EA jump (left) and DE EA jump (right) respectively. Parameters: $\nu=4.04, \vartheta=0.05, \sigma_{0}=$ $1.01, \rho \in\{0,-0.54\}, \zeta=0.03, \sigma_{e}=.0473, u=0.5, \eta_{1}=\eta_{2}=40$.

\subsection{Small and Large Strikes Asymptotics}

We now analyze the asymptotics of the IV surface for small and large strikes for the extended Heston and Kou model. Our asymptotics follow from an adaptation of the results of Benaim and Friz (2008) (see also Benaim et al. (2012)). We state the results here and provide the proofs in Appendix A.4.

Proposition 4.4. Let $S$ satisfy the extended Heston dynamics (3.3) where $Z_{e}$ is either:

Case 1: normally distributed, $Z_{e} \sim N\left(0, \frac{\sigma_{e}^{2}}{2}\right)$; or

Case 2: double-exponentially distributed, $Z_{e} \sim D E\left(u, \eta_{1}, \eta_{2}\right)$. 
Then for any fixed $t<T_{e}$, the implied volatility $I(t ; K, T)$ satisfies

$$
\begin{aligned}
& \frac{I^{2}(t ; K, T)(T-t)}{\log \left(\frac{K}{S_{t}}\right)} \sim \xi\left(q^{*}\right), \text { as } K \rightarrow 0, \\
& \frac{I^{2}(t ; K, T)(T-t)}{\log \left(\frac{K}{S_{t}}\right)} \sim \xi\left(r^{*}-1\right), \text { as } K \rightarrow \infty,
\end{aligned}
$$

where $\xi(x)$ is defined by $\xi(x) \equiv 2-4\left(\sqrt{x^{2}+x}-x\right)$ and

$$
q^{*}=\left\{\begin{array}{ll}
p_{-} & \text {case } 1, \\
\min \left\{p_{-}, \eta_{2}\right\} & \text { case } 2,
\end{array} \quad, \quad r^{*}= \begin{cases}p_{+} & \text {case } 1, \\
\min \left\{p_{+}, \eta_{1}\right\} & \text { case } 2,\end{cases}\right.
$$

and $p_{ \pm}$is the smallest positive solution to, respectively,

$$
\nu \mp \rho \zeta p_{ \pm}+\sqrt{\left(\nu \mp \rho \zeta p_{ \pm}\right)^{2}+\zeta^{2}\left( \pm p_{ \pm}-\left(p_{ \pm}\right)^{2}\right)} \operatorname{coth}\left(\frac{(T-t)}{2} \sqrt{\left(\nu \mp \rho \zeta p_{ \pm}\right)^{2}+\zeta^{2}\left( \pm p-\left(p_{ \pm}\right)^{2}\right)}\right)=0 .
$$

Proposition 4.5. Let $S$ follow the extended Kou dynamics (3.1) where $Z_{e}$ is either:

Case 1: normally distributed, $Z_{e} \sim N\left(0, \frac{\sigma_{e}^{2}}{2}\right)$; or

Case 2: double-exponentially distributed, $Z_{e} \sim D E\left(u, \eta_{1}, \eta_{2}\right)$.

Then for any fixed $t<T_{e}$, the implied volatility $I(t ; K, T)$ satisfies

$$
\begin{aligned}
& \frac{I^{2}(t ; K, T)(T-t)}{\log \left(\frac{K}{S_{t}}\right)} \sim \xi\left(q^{*}\right), \text { as } K \rightarrow 0, \\
& \frac{I^{2}(t ; K, T)(T-t)}{\log \left(\frac{K}{S_{t}}\right)} \sim \xi\left(r^{*}-1\right), \text { as } K \rightarrow \infty,
\end{aligned}
$$

where $\xi(x) \equiv 2-4\left(\sqrt{x^{2}+x}-x\right)$ and

$$
q^{*}=\left\{\begin{array}{ll}
\lambda_{2} & \text { case } 1, \\
\min \left\{\lambda_{2}, \eta_{2}\right\} & \text { case } 2,
\end{array} \quad, \quad r^{*}= \begin{cases}\lambda_{1} & \text { case } 1, \\
\min \left\{\lambda_{1}, \eta_{1}\right\} & \text { case } 2\end{cases}\right.
$$

First, we observe that if the EA jump is Gaussian, then it has no role in the IV asymptotics in strikes (see (4.7) and (4.9)). Hence, in either the Heston or Kou model, the large/small strikes asymptotics with and without the EA jump are in fact identical. On the other hand, if the tails of the EA jump are fatter than those of the base model, then the IV asymptotics are determined by the EA jump parameters. In such cases, the asymptotics are observed for less extreme strikes with short maturities, which is when the EA jump variance dominates. For longer maturities, however, the asymptotics hold for more extreme strikes. An intuitive explanation is that, as time-to-maturity increases, the EA jump variance is relatively low and the tails behavior is manifest only for extreme values.

In Figure 7, we show the IV asymptotics under the extended Kou (left) and Heston (right) models compared to the IV obtained by inverting the BS formula on option prices calculated via Fourier transform (see Section 3.2). We plot the asymptotic volatility function $I(t ; K, T)=c+\sqrt{\log \left(K / S_{t}\right) \xi(\omega)}$ for fixed $(t, T)$, where $c$ is a constant chosen so that the asymptotics and the model IVs coincide at the most extreme strikes considered. In each particular case, $\omega$ is a constant set according to (4.9)-(4.10) (for the 
extended Kou model) and (4.7)-(4.8) (for the extended Heston model). In Figure 7 (left) the doubleexponential EA jump tails dominates those of the daily jumps, as this generally holds in practice. In Figure 7 (right), the EA jump is Gaussian and thus does not affect the IV asymptotics from the base model.
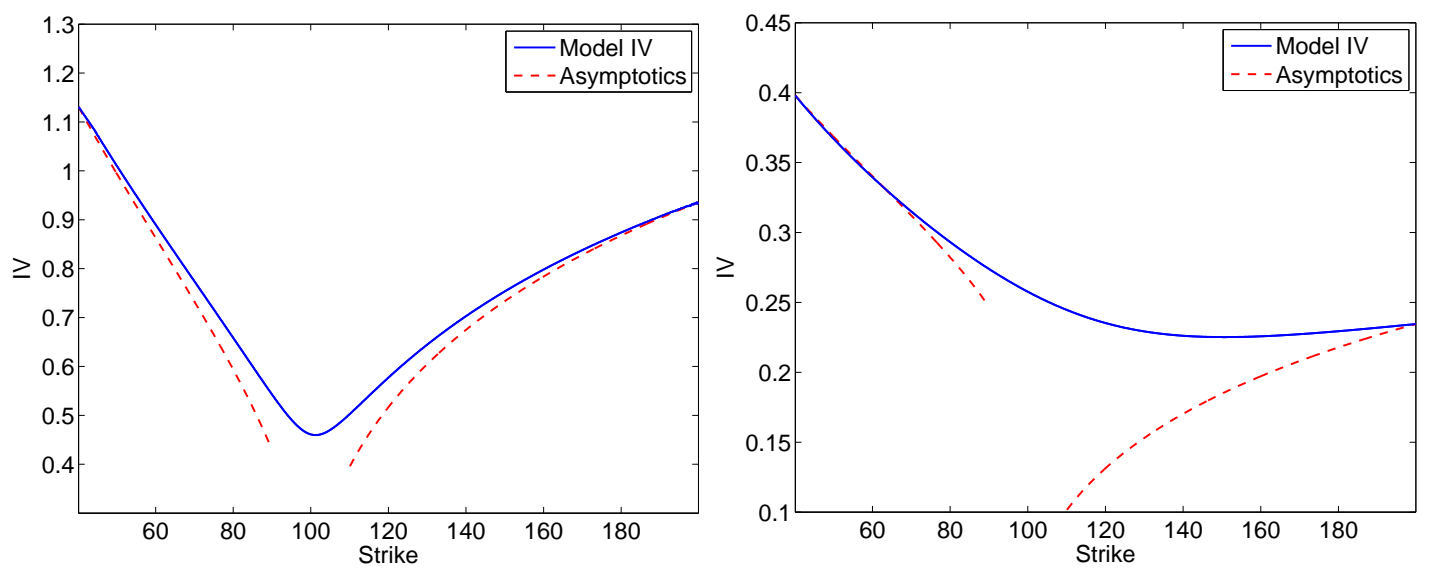

Figure 7: (Left) The IV obtained from numerical option prices against the asymptotics (4.9)-(4.10) under the extended Kou model with a log-DE EA jump. Parameters: $S=100, r=0.02, T=\frac{4}{252}, \kappa=300, p=$ $0.5, \lambda_{1}=\lambda_{2}=100, u=0.5, \eta_{1}=30, \eta_{2}=25$. (Right) The IV obtained from numerical option prices against the asymptotics (4.7)-(4.8) under the extended Heston model with a log-normal EA jump. Parameters: $S=100, r=0.02, T=1, \nu=2.7, \vartheta=0.077, \sigma_{0}=0.7075, \rho=-0.54, \zeta=0.073, \sigma_{e}=0.04$.

\section{Calibration and Parameter Estimators}

In this section, we perform calibrations of the extended BS, Kou and Heston models to the observed market prices of options near an earnings announcement. This allows us to evaluate whether the model extensions improve the accuracy of the calibration compared to that of the base models. Calibration results can also be used to infer information about the distribution of the EA jump and, in one simple example, we compare the estimators obtained through calibration on IBM options data to estimates given by its historical distribution.

\subsection{Model Calibration}

In our calibration procedure, we consider a set of $N$ vanilla calls and puts with observed market prices, $\hat{C}_{i}, i=1, \ldots, N$. These options have different contractual features, such as strike, maturity, and option type. For a given model, the set of model parameters is denoted by $\Theta$. In turn, the model price of option $i$ is denoted by $C_{i}(\Theta)$. To calibrate a given model, we minimize the sum of squared errors (see e.g. Dennis (1977); Andersen and Andreasen (2000); Bates (1996); Cont and Tankov (2002)):

$$
\min _{\Theta} \sum_{i=1}^{N}\left(C_{i}(\Theta)-\hat{C}_{i}\right)^{2}
$$

We use the best-bid and best-ask mid-point prices. Furthermore, we adapt a trust-region-reflective gradient-descent algorithm (Coleman and Li (1994); Coleman and Li (1996)), starting from different initial points to guarantee a better exploration of the parameters space. While our numerical tests show 
the adopted method results in an effective calibration, we remark that there are many alternative, possibly more advanced, calibration procedures available (see e.g. Cont and Tankov (2002) and references therein).

The majority of equity options in the US are of the American type. While in Section 6 we will discuss the pricing of American options, the methods are generally too computationally-intensive to be practically used in conjunction with a gradient-descent method for calibration, especially as the number of options and parameters increase. Related studies typically circumvent this issue by simply assuming the American options are European-style (see e.g. Dubinsky and Johannes (2006); Broadie et al. (2009)). In contrast, our procedure begins by inverting the market prices of American puts and calls via a relatively fast American option pricer under the Black-Scholes model. This gives us the observed IVs. In turn, we apply the Black-Scholes European put/call pricing formula, with the volatility parameter being the observed IV, and derive the associated European put or call price. We then use the resulting prices as inputs to calibrate against the option prices generated from a model. In all our experiments, we obtain option price data, available up to August 2013, from the OptionMetrics Ivy database.

We now present an example using the extended BS, Kou and Heston models with different distributions for the EA jump. Our objective is to illustrate the calibrated IV surfaces under these models and compare them with the empirical IV surface. Recall from Figure 2 the empirical implied volatility surface of IBM on July 15, 2013. That is observed 2 days prior to the earnings announcement by IBM on July 17, 2013 after market. The closest options expiration date was Friday July 19, 2013. As noted earlier, the front month IVs are significantly higher than those for options with longer maturities. In Figure 8 we show the associated calibrated IV surfaces for the 3 base models and their extensions, resulting in a total of 9 calibrated models. Table 2 summarizes the calibrated parameters. In the original Black-Scholes model, the implied volatility surface is flat and takes a high value of $28.11 \%$. As we incorporate the EA jump, under both Gaussian and DE distributions, the calibrated values of the stock price volatility $\sigma$ are lower. More importantly, in every case, the base model is unable to generate the characteristic shape of the IV surface before the EA. Between the extended Heston and Kou models, the IV surface generated from the Kou model tends to flatten more rapidly as maturity lengthens. Overall, the Heston model seems to be able to reproduce the IV surface more accurately, and the incorporation of a Gaussian EA jump seems to reproduce the IV surface better than with a DE EA jump in this example. We will further compare the two EA jump distributions in Section 5.3 .

\begin{tabular}{|c|c|c|c|c|c|c|c|c|c|}
\hline Black-Scholes & $\sigma$ & $\sigma_{e}$ & $\mathrm{u}$ & $\eta_{1}$ & $\eta_{2}$ & & & & \\
\hline Base & $28.11 \%$ & - & - & - & - & & & & \\
\hline Gaussian jump & $27.69 \%$ & $7.11 \%$ & - & - & - & & & & \\
\hline DE jump & $21.42 \%$ & - & $38.28 \%$ & 23.03 & 6.3 & & & & \\
\hline Heston & $\nu$ & $\vartheta$ & $\zeta$ & $\rho$ & $\sigma_{0}^{2}$ & $\sigma_{e}$ & $\mathrm{u}$ & $\eta_{1}$ & $\eta_{2}$ \\
\hline Base & 3.70 & 0.05 & 0.90 & -0.51 & 0.05 & - & - & - & - \\
\hline Gaussian jump & 4.04 & 0.05 & 1.01 & -0.55 & 0.03 & $4.73 \%$ & - & - & - \\
\hline DE jump & 3.10 & 0.05 & 0.84 & -0.54 & 0.03 & - & $42.06 \%$ & 34.77 & 24.95 \\
\hline Kou & $\sigma$ & $\kappa$ & $\mathrm{p}$ & $\lambda_{1}$ & $\lambda_{2}$ & $\sigma_{e}$ & $\mathrm{u}$ & $\eta_{1}$ & $\eta_{2}$ \\
\hline Base & $2.80 \%$ & 232.3 & $46.85 \%$ & 275.9 & 82.1 & - & $\overline{-}$ & $\overline{-}$ & 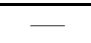 \\
\hline Gaussian jump & $0.03 \%$ & 193.6 & $51.17 \%$ & 998.9 & 70.0 & $3.61 \%$ & - & - & - \\
\hline DE jump & $0.39 \%$ & 85.1 & $79.46 \%$ & 990.3 & 30.0 & - & $98.73 \%$ & 32.9 & 2.0 \\
\hline
\end{tabular}

Table 2: Summary of calibrated parameters based on the observed IV surface in Figure 2, The corresponding calibrated IV surfaces are displayed in Figure 8 

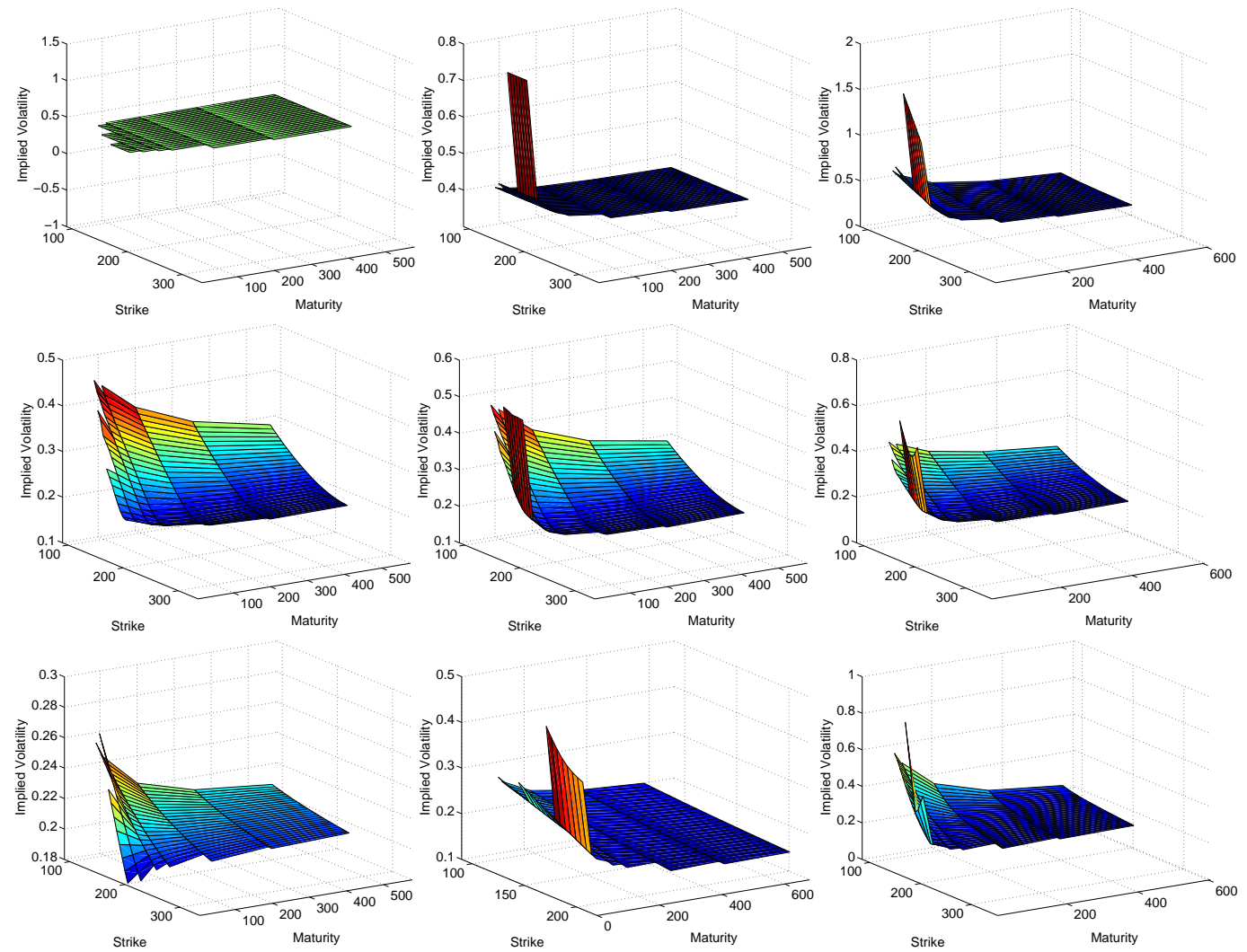

Figure 8: Calibrated surfaces for the Black-Scholes, Heston, and Kou models (1st, 2nd, 3rd rows respectively) without EA jump (left column), and with Gaussian (middle) and DE (right) EA jumps. The calibrated model parameters are listed in Table 2 .

\subsection{Analytic Estimators under the Extended BS Model}

In the extended BS model (2.1), it is possible to derive analytical estimators for the models parameters $\left(\sigma, \sigma_{e}\right)$, as discussed in Dubinsky and Johannes (2006). We apply these estimators to compare with the estimators obtained by calibrating other models (see Table 3). First, we consider the extended BS model where the EA jump $Z_{e}$ is normally distributed. To calibrate this model, it suffices to use any pair of options of different maturities. Let $\sigma_{I V}\left(T_{1}\right), \sigma_{I V}\left(T_{2}\right)$ represent the implied volatilities of two options with maturities $T_{1}$ and $T_{2}$, respectively. Then, applying (2.4), the model parameters $\left(\sigma, \sigma_{e}\right)$ can be estimated by

$$
\sigma^{T S}=\sqrt{\frac{\left(T_{1}-t\right) \sigma_{I V}^{2}\left(T_{1}\right)-\left(T_{2}-t\right) \sigma_{I V}^{2}\left(T_{2}\right)}{T_{1}-T_{2}}}, \quad \sigma_{e}^{T S}=\sqrt{\frac{\sigma_{I V}^{2}\left(T_{1}\right)-\sigma_{I V}^{2}\left(T_{2}\right)}{\frac{1}{T_{1}-t}-\frac{1}{T_{2}-t}}} .
$$

where the superscript TS indicates the relevance of the IV term structure to these estimators. In particular, we call $\sigma_{e}^{T S}$ the term structure estimator of the jump volatility under the risk-neutral measure $\mathbb{Q}$. We stress that a set of two options with identical maturity would not allow us to estimate $\sigma$ and $\sigma_{e}$ separately, but only the aggregate value $\sigma^{2}+\frac{\sigma_{e}^{2}}{T-t}$.

Alternatively, one can utilize option prices at different times for parameter estimation. In fact, given the implied volatilities $\sigma_{I V, t_{1}}$ and $\sigma_{I V, t_{2}}$ at times $t_{1}$ and $t_{2}$, with $t_{1}<t_{2}<T_{e}$, we apply (2.4) to get the 
following estimators:

$$
\sigma^{t s}=\sqrt{\frac{\left(T-t_{1}\right) \sigma_{I V, t_{1}}^{2}(T)-\left(T-t_{2}\right) \sigma_{I V, t_{2}}^{2}(T)}{t_{2}-t_{1}}}, \quad \sigma_{e}^{t s}=\sqrt{\frac{\sigma_{I V, t_{1}}^{2}(T)-\sigma_{I V, t_{2}}^{2}(T)}{\frac{1}{T-t_{1}}-\frac{1}{T-t_{2}}}} .
$$

They are called the time-series estimators (see also Dubinsky and Johannes (2006)).

We observe from (5.2) that one must require that $\sigma_{I V}\left(T_{1}\right)>\sigma_{I V}\left(T_{2}\right)$ in order to obtain well-defined estimators. Similarly for $\sigma^{t s}$ and $\sigma_{e}^{t s}$ in (5.3) , their definitions suggest that $\sigma_{I V, t_{2}}>\sigma_{I V, t_{1}}$ must hold. In our empirical tests, we find that, before an earnings announcement, $\sigma_{I V}\left(T_{1}\right)>\sigma_{I V}\left(T_{2}\right)$ always holds, but the condition $\sigma_{I V . t_{1}}>\sigma_{I V, t_{2}}$ is sometimes violated. Similar observations are also discussed in Dubinsky and Johannes (2006), who have also conducted a comprehensive empirical test using ATM options.

We emphasize that these analytical estimators are based on a specific extension of the BS model. Since market prices are not necessarily generated by this model, the analytical estimators and the calibrated parameters may not coincide. Moreover, they also depend on the choice of options whose IVs are inputs to the estimator formulas. On the other hand, the main advantage of these analytical estimators is that they can be computed instantly, and they are also used in practice (see e.g. Mehra et al. (2014)) and related studies.

\subsection{Implied EA jump Distribution and Risk Premia}

With the choice of a pricing model, our calibration procedure extracts the implied distribution of the EA jump. One useful application is to compare the risk-neutral and historical distributions of the EA jump. Their discrepancy will shed some light on the risk premium associated with the EA jump. As an example, let us consider the empirical EA jumps of the IBM stock starting from 1994. We assume that both risk-neutral and historical distributions are Gaussian, which is amenable for comparison since we only need to estimate a single parameter, i.e. the EA jump volatility. In Table 3, we report the estimate of EA jump volatility obtained by calibrating the Heston model extended with a Gaussian EA jump, $\sigma_{e}^{Q}$, and the empirical EA jump volatility, $\sigma_{e}^{P}$ based on data from 1994 up to the given date. For comparison, we also list the EA jump volatility estimators according to (5.2). As we can see, for each given date, the ratio $\sigma_{e}^{P} / \sigma_{e}^{Q}$ is very close to 1 . This suggests that under the extended Heston model, the EA jump distributions are very similar under both historical and risk-neutral measures. On the other hand, the ratio $\sigma_{e}^{P} / \sigma_{e}^{T S}$ is smaller and less than 1 in this example, suggesting that the extended BS model would imply a higher EA jump volatility than the empirical one. In summary, the volatility $\sigma_{e}^{Q}$ calibrated from the extended Heston model is smaller than the term structure EA jump volatility estimator $\sigma_{e}^{T S}$ which is based on the extended BS model without stochastic volatility.

\begin{tabular}{rrrrrr}
\hline Date & $\sigma_{e}^{P}$ & $\sigma_{e}^{Q}$ & $\sigma_{e}^{P} / \sigma_{e}^{Q}$ & $\sigma_{e}^{T S}$ & $\sigma_{e}^{P} / \sigma_{e}^{T S}$ \\
\hline 18-Jul-12 & $4.64 \%$ & $4.68 \%$ & $99.3 \%$ & $5.22 \%$ & $89.0 \%$ \\
16-Oct-12 & $4.62 \%$ & $4.18 \%$ & $110.5 \%$ & $4.70 \%$ & $98.4 \%$ \\
22-Jan-13 & $4.62 \%$ & $4.58 \%$ & $100.9 \%$ & $5.71 \%$ & $81.0 \%$ \\
18-Apr-13 & $4.61 \%$ & $4.68 \%$ & $98.5 \%$ & $5.49 \%$ & $84.1 \%$ \\
17-Jul-13 & $4.63 \%$ & $4.61 \%$ & $100.4 \%$ & $5.76 \%$ & $80.3 \%$ \\
\hline
\end{tabular}

Table 3: The implied and historical EA jump volatilities for IBM. For each date in the table, the historical EA jump volatility $\sigma_{e}^{P}$ is estimated using price data from 1994 up to that date. The implied volatility $\sigma_{e}^{Q}$ is calibrated from the extended Heston model. 


\section{American Options}

While index options are typically of European style, most US equity options are American-style. In general, the American option pricing problem does not admit closed-form formulas, so we discuss a numerical method for computing the option price and exercise boundary. In addition, we apply the analytic results from the European case to approximate the American option price before an earnings announcement.

\subsection{American Option Price and Exercising Boundary}

We assume that the stock price evolves according to the extended Kou model with an EA jump defined in (3.1). The value of the American option is defined by

$$
A(t, S)=\sup _{t \leq \tau \leq T} \mathbb{E}\left\{e^{-r(\tau-t)}\left(K-S_{\tau}\right)^{+} \mid S_{t}=S\right\}, \quad t \leq T
$$

where $\tau$ is a stopping time w.r.t. the filtration generated by $S$. By the dynamic programming principle, the option price can be written as (see, e.g. (Øksendal, 2003, Chap. 10))

$$
A(t, S)=\sup _{t \leq \tau \leq T_{e}} \mathbb{E}\left\{e^{-r(\tau-t)}\left(1_{\left\{\tau<T_{e}\right\}}\left(K-S_{\tau}\right)^{+}+1_{\left\{\tau=T_{e}\right\}} A\left(T_{e}, S_{T_{e}-} e^{Z_{e}}\right)\right) \mid S_{t}=S\right\}
$$

Therefore, we see that for $t<T_{e}$ the problem is equivalent to pricing an American option under the Kou model but with "terminal" payoff $\mathbb{E}\left\{A\left(T_{e}, S e^{Z_{e}}\right) \mid S_{T_{e-}}=S\right\}$ at time $T_{e}$. Assume that $t<T_{e}<T$ and that $Z_{e}$ admits the p.d.f. $g(z)$. Then, the American put price can be written as

$$
A(t, S)=D(t, S) 1_{\left\{t<T_{e}\right\}}+\widetilde{D}(t, S) 1_{\left\{t \geq T_{e}\right\}}
$$

where $D$ and $\widetilde{D}$ satisfy the linear complementarity problems (see Bensoussan and Lions (1984) and Cont and Voltchkova (2005)):

$$
\begin{aligned}
& \begin{cases}\widetilde{D}(t, S) \geq(K-S)^{+}, & T_{e} \leq t<T, S \geq 0 \\
r \widetilde{D}(t, S)-\frac{\partial \widetilde{D}}{\partial t}(t, S)-\mathcal{L} \widetilde{D}(t, S) \geq 0, & T_{e} \leq t<T, S \geq 0 \\
\left(\widetilde{D}(t, S)-(K-S)^{+}\right)\left(r \widetilde{D}(t, S)-\frac{\partial \widetilde{D}}{\partial t}(t, S)-\mathcal{L} \widetilde{D}(t, S)\right)=0, & T_{e} \leq t<T, S \geq 0 \\
\widetilde{D}(T, S)=(K-S)^{+}, & S \geq 0\end{cases} \\
& \begin{cases}D(t, S) \geq(K-S)^{+}, & 0 \leq t<T_{e}, S \geq 0 \\
r D(t, S)-\frac{\partial D}{\partial t}(t, S)-\mathcal{L} D(t, S) \geq 0, & 0 \leq t<T_{e}, S \geq 0 \\
\left(D(t, S)-(K-S)^{+}\right)\left(r D(t, S)-\frac{\partial D}{\partial t}(t, S)-\mathcal{L} D(t, S)\right)=0, & 0 \leq t<T_{e}, S \geq 0 \\
D\left(T_{e}, S\right)=\int_{\mathbb{R}} \widetilde{D}\left(T_{e}, S e^{z}\right) g(z) d z, & S \geq 0\end{cases}
\end{aligned}
$$

We have denoted by $\mathcal{L}$ the infinitesimal generator of $S$ under model (3.1):

$$
\mathcal{L} V(S) \equiv \frac{\sigma^{2} S^{2}}{2} \frac{\partial^{2} V}{\partial S^{2}}+r S \frac{\partial V}{\partial S}+\kappa \int_{-\infty}^{\infty}\left(V\left(S e^{y}\right)-V(S)\right) f_{J}(y) d y
$$

and $f_{J}$ is double exponential p.d.f.

It is worth noting that the integration at time $T_{e}$ in (6.4) must be approximated with a sum because $\tilde{D}$ is not in closed form. The numerical computation of the integration may introduce computational errors, but it also adds to the computational burden since the sum is $\mathcal{O}\left(n^{2}\right)$, where $n$ is the number of 
discretized stock price values. We remark that it is possible, for example, to reduce the complexity of the integration to $\mathcal{O}(n \log (n))$ using an FFT algorithm. It is also worth noticing that when $T_{e}=T$, the complexity reduces to $\mathcal{O}(n)$ if closed form formulas are available for the European option (e.g. if the EA jump is Gaussian or double-exponential). On the other hand, the complexity also reduces to $\mathcal{O}(n)$ when the announcement is imminent, i.e. $T_{e}=0^{+}$. This motivates us to look for a closed-form approximation to the American option price based on these scenarios, as discussed in Sect. 6.2. It is useful to compare the American option prices under the same model but with different earnings announcement dates.

Proposition 6.1. Let $A(t, S ; u)$ denote the American option price as in (6.1) with $T_{e}=u$. Then, we have

$$
A(t, S ; l) \leq A(t, S ; u) \quad, \quad l \geq u>t .
$$

As a result, the American option price is monotonically decreasing as the EA date $T_{e}$ approaches maturity. Consequently, $A(t, S ; t)$ and $A(t, S ; T)$ become the upper and lower bounds, respectively, for the American option price $A(t, S ; u), t \leq u \leq T$. As an interesting comparison, the European option price is completely independent of the exact EA date as long as it is at or prior to maturity. In Figure 9 (right \& top) we show the time-value 3 of an American put, with strike, maturity, and spot price fixed, over different announcement times $T_{e}$. Consistent with Proposition 6.1, the time-value of the American put is indeed monotonically decreasing in $T_{e}$.

To solve problems (6.3)-(6.4) we use the Fourier transform based method presented in Jackson et al. (2008). Unless $T_{e}=T$ or $T_{e}=0^{+}$, we solve backward in time for $\tilde{D}$ in (6.3), perform the numerical integration and feed it as a terminal condition for problem (6.4) which is solved backward in time as well.

Figure 9 (left) shows the exercise boundary for different values of $T_{e}$ under the Kou model with a DE EA jump, along other common parameters. Naturally, the scheduled announcement introduces a discontinuity in the exercising boundary. We mark the EA dates with three crosses. As expected, after the largest date $T_{e}$ the three boundaries coincide. Interestingly, the exercise boundary is decreasing rapidly in time near $T_{e}$, which means that the option holder is more likely to wait until the earnings announcement, rather than exercising immediately. This can also be seen in terms of the option's time-value. In Figure 9 (right \& bottom), we illustrate that the time-value of an American put, with both strike and spot price fixed, is increasing as time approaches the EA date.

\subsection{Analytical Approximations}

One major method proposed by Barone-Adesi and Whalev (1987) to analytically approximate the price of an American option is to express the American option price in terms of an European option price plus a correction term. The correction term is determined as the solution of an approximation of the BlackScholes equation, with the addition of elementary boundary condition. Kou and Wang (2004) present an analytical approximation to the American option price under the Kou model. Here, we adapt the Barone-Adesi approximation and apply it to our extended Kou model.

When $T_{e}=T$ the approximation is virtually identical to the original one. Let $P_{E}(t, S)$ denote the European put price (as in Proposition 3.1). The approximate price for the American put is similar to that of Kou and Wang (2004) and is given by

$$
\tilde{A}(t, S)= \begin{cases}P_{E}(t, S)+\gamma_{1} S^{-\beta_{1}}+\gamma_{2} S^{-\beta_{2}} & , \text { if } S>\alpha(t), \\ (K-S)^{+} & , \text {if } S \leq \alpha(t)\end{cases}
$$

\footnotetext{
${ }^{3}$ The time-value of an American put is defined as $A-(K-S)^{+}$where $A$ is the put price, $S$ is the spot price and $K$ is the strike. With spot price $S$ fixed, inequality (6.6) also holds for the corresponding time-values.
} 

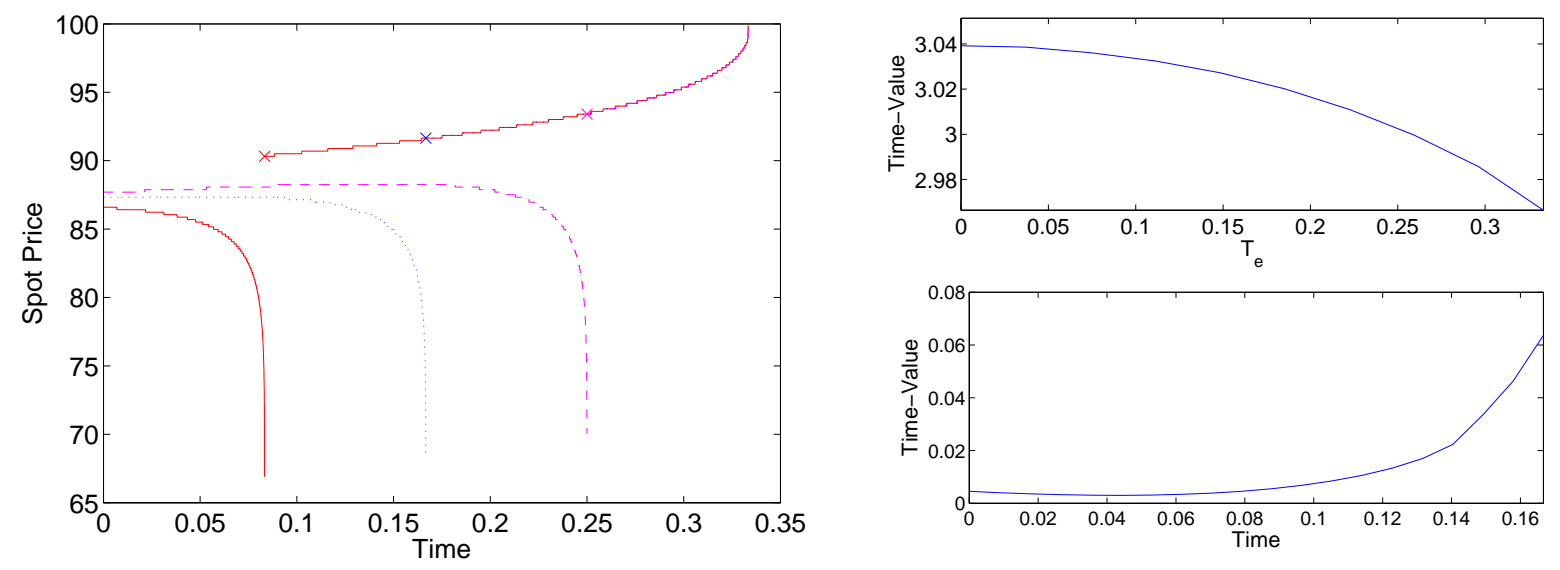

Figure 9: Left: The American put exercising boundary when $T_{e}$ assumes different values, $T_{e}=1,2,3$ months. Right: The time-value of a put (top) with spot and strike set at $\$ 99.79$ when $T_{e}$ changes and the time-value of a put (bottom) with strike $\$ 100$ and spot $\$ 87.33$ when $T_{e}=2$ months and time $t$ changes. Parameters: $r=0.02, T=4$ months, $\sigma=0.1, \kappa=252, p=0.5, \lambda_{1}=300, \lambda_{2}=300, u=0.5, \eta_{1}=30, \eta_{2}=30$

with the positive constants

$$
\begin{aligned}
& \gamma_{1} \equiv \frac{\alpha^{\beta_{1}}}{\beta_{2}-\beta_{1}}\left(\beta_{2} K-\left(1+\beta_{2}\right)\left(\alpha+P_{E}(t, \alpha)\right)+K e^{-r(T-t)} q(\alpha(t))\right), \\
& \gamma_{2} \equiv \frac{\alpha^{\beta_{2}}}{\beta_{1}-\beta_{2}}\left(\beta_{1} K-\left(1+\beta_{1}\right)\left(\alpha+P_{E}(t, \alpha)\right)+K e^{-r(T-t)} q(\alpha(t))\right),
\end{aligned}
$$

where $q(s) \equiv \mathbb{Q}\left\{S_{T} \leq K \mid S_{t}=s\right\}$, and $\beta_{1,2}, 0<\beta_{1}<\lambda_{2}<\beta_{2}<\infty$, are the two positive solutions to the equation

$$
\frac{r}{1-e^{-r(T-t)}}=\beta\left(m \kappa-\frac{\sigma^{2}}{2}-r\right)+\frac{\sigma^{2} \beta^{2}}{2}+\kappa\left(p \frac{\lambda_{1}}{\lambda_{1}+\beta}+(1-p) \frac{\lambda_{2}}{\lambda_{2}-\beta}-1\right)
$$

Also, $\alpha(t) \in[0, K]$ is the solution to the equation

$$
c_{1} K-c_{2}\left(\alpha(t)+P_{E}(t, \alpha(t))\right)=\left(c_{1}-c_{2}\right) K e^{-r(T-t)} q(\alpha(t))
$$

with $c_{1}=\beta_{1} \beta_{2}\left(1+\lambda_{2}\right)$ and $c_{2}=\lambda_{2}\left(1+\beta_{1}\right)\left(1+\beta_{2}\right)$. The analytical expression for $P_{E}(t, S)$ and $q(s)$ can be directly obtained from Proposition 3.1. In contrast to the formula given in Kou and Wang (2004) (see equation (7) there), the calculation of $P_{E}(s, t), q(\alpha(t)), \gamma_{1,2}$ and $\alpha$ accounts for the EA jump r.v. $Z_{e}$ (see Appendix A.6).

When $t \leq T_{e}<T$, one could apply the same method used to derive the approximation above, writing the American option as $\tilde{A}(t, S)=\mathbb{E}\left\{e^{-r\left(T_{e}-t\right)} \tilde{A}\left(T_{e}, S_{T_{e}}\right) \mid S_{t}=S\right\}+\epsilon(t, S)$. The functional form of $\epsilon$ would be identical, $\epsilon(t, S)=\gamma_{1} S^{-\beta_{1}}+\gamma_{2} S^{-\beta_{2}}$, because it is derived from the same PIDE (see Appendix A.6). The constants $\beta_{1}$ and $\beta_{2}$ are indeed solutions to equation (6.10), after adjusting the time parameters accordingly. In the case when the EA jump is imminent $\left(T_{e}=t^{+}\right)$, we need to evaluate the expectation $\mathbb{E}\left\{\tilde{A}\left(t_{+}, S e^{Z_{e}}\right)\right\}$, where $S$ is the stock price at time $t$. From (6.7), this amounts to computing

$$
\int_{-\infty}^{+\infty}\left(\left(P_{E}\left(t_{+}, S e^{z}\right)+\gamma_{1} e^{-\beta_{1} z} S^{-\beta_{1}}+\gamma_{2} e^{-\beta_{2} z} S^{-\beta_{2}}\right) 1_{\left\{S e^{z}>\alpha\right\}}+\left(K-S e^{z}\right) 1_{\left\{S e^{z} \leq \alpha\right\}}\right) f_{Z_{e}}(z) d z
$$




\begin{tabular}{r|rrrrrrrrrrrr}
\hline $\mathrm{K} \backslash T_{e}$ & $T-2 \mathrm{D}$ & $1.5 \mathrm{M}$ & \multicolumn{1}{c}{$3 \mathrm{D}$} & $T$ & 0 & - & $T-2 \mathrm{D}$ & $1.5 \mathrm{M}$ & $3 \mathrm{D}$ & $T$ & 0 \\
\hline & FST & FST & FST & $B A_{L}$ & $B A_{H}$ & EU & FST & FST & FST & $B A_{L}$ & $B A_{H}$ & EU \\
\hline 80 & 0.10 & 0.10 & 0.10 & 0.10 & 0.11 & 0.10 & 0.01 & 0.01 & 0.01 & 0.01 & 0.01 & 0.01 \\
85 & 0.37 & 0.37 & 0.37 & 0.37 & 0.37 & 0.37 & 0.05 & 0.05 & 0.05 & 0.05 & 0.05 & 0.05 \\
90 & 1.02 & 1.02 & 1.02 & 1.02 & 1.02 & 1.02 & 0.22 & 0.22 & 0.23 & 0.22 & 0.23 & 0.22 \\
95 & 2.30 & 2.31 & 2.31 & 2.30 & 2.31 & 2.29 & 0.84 & 0.86 & 0.87 & 0.84 & 0.87 & 0.84 \\
100 & 4.40 & 4.42 & 4.43 & 4.40 & 4.42 & 4.38 & 2.54 & 2.60 & 2.63 & 2.54 & 2.62 & 2.53 \\
105 & 7.35 & 7.39 & 7.40 & 7.36 & 7.38 & 7.32 & 5.68 & 5.81 & 5.91 & 5.70 & 5.91 & 5.66 \\
110 & 11.07 & 11.11 & 11.14 & 11.06 & 11.11 & 10.98 & 10.01 & 10.10 & 10.28 & 10.03 & 10.30 & 9.87 \\
115 & 15.36 & 15.40 & 15.45 & 15.35 & 15.42 & 15.20 & 15.00 & 15.00 & 15.08 & 15.00 & 15.10 & 14.57 \\
120 & 20.06 & 20.07 & 20.14 & 20.04 & 20.13 & 19.77 & 20.00 & 20.00 & 20.01 & 20.00 & 20.04 & 19.45 \\
\hline
\end{tabular}

Table 4: American put prices under the extended Kou model with a DE EA jump. The column "FST" shows the prices calculated by a Fourier transform method for three different values of $T_{e}$ with expiration date $T=3$ months fixed. The extended Barone-Adesi approximations (6.7) and (6.12) are given under columns " $B A_{L}$ " and " $B A_{U}$ ". Column "EU" shows the corresponding European put price (see (3.2)). The first 6 columns are calculated with model parameters: $S=100, r=0.02, \sigma=0.2, \kappa=252, p=0.5, \lambda_{1}=300, \lambda_{2}=300, u=0.5, \eta_{1}=30, \eta_{2}=30$. The last 6 columns with $S=100, r=0.02, \sigma=0.07, \kappa=200, p=0.5, \lambda_{1}=350, \lambda_{2}=350, u=0.5, \eta_{1}=25, \eta_{2}=25$.

where $\alpha, \gamma_{1,2}$, and $\beta_{1,2}$ are determined by (6.8)-(6.11). Notice that at time $t$ there is no exercising boundary since $\tilde{A}(t, S) \geq(K-S)^{+}$due to Jensen's inequality. In the extended Kou model with doubleexponential EA jump, semi-closed formulas can be obtained in a similar fashion as in Proposition 3.1 .

In Table 4, we present numerical results for the prices of American put options with different strikes and maturities under the extended Kou model. We compare the price computed from the Fourier transform method to that analytical approximations (6.7) and (6.12). For different choices of model parameters, strikes and maturities, the approximations perform very well when $T_{e} \sim t$ or $T_{e} \sim T$. For the second set of parameters (last 6 columns), the EA jump has a greater impact on the options prices as the tails of the jump are fatter, and the volatility of the other part of the dynamics is lower. In this case, the difference between the "true" prices for different values of $T_{e}$ increases, and so does the difference between the two approximations which might then not be suitable to approximate the options values for in-the-money options when $T_{e}$ is not close to $t$ or $T$.

\section{A Appendix}

In this Appendix, we provide a number of detailed proofs and formulas.

\section{A.1 Details for Formula (3.2)}

In this section we write the expression of the function $\Upsilon$ in (3.2) explicitly. For ease of notation, we refer to the parameters given as input to $\Upsilon$ as a vector $\Theta \equiv\left(\theta_{1}, \ldots, \theta_{13}\right)$. In addition, let $\tilde{\Theta}$ be a permutation of the vector $\Theta$ where only the 8th and 9th components (the parameters for the randomly timed jumps) are switched. The function $\Upsilon$ is given by

$$
\Upsilon(\Theta)=\sum_{n=0}^{\infty} \frac{\left(\theta_{6} \theta_{4}\right)^{n} e^{-\theta_{6} \theta_{4}}}{n !} Z_{n}(\Theta)
$$

where

$$
Z_{n}(\Theta)=\theta_{10} \sum_{l=0}^{n}\left(P_{n, l} T_{1, n}(k, \Theta)+Q_{n, l} T_{2, n}(k, \Theta)\right)+\theta_{11} \sum_{l=0}^{n}\left(P_{n, l} T_{3, n}(k, \Theta)+Q_{n, l} T_{4, n}(k, \Theta)\right),
$$




$$
\begin{aligned}
& k=\log \left(\frac{\theta_{2}}{\theta_{1}}\right)-\left(\theta_{3}-\frac{\theta_{5}^{2}}{2}-m \kappa\right) \theta_{4}-\alpha, \quad m=p \frac{\lambda_{1}}{\lambda_{1}-1}+q \frac{\lambda_{2}}{\lambda_{2}+1}-1 \\
& T_{1, n+1}(s, \Theta)=\frac{\theta_{8}}{\theta_{8}-\theta_{12}}\left(T_{1, n}(s, \Theta)-\theta_{12} \frac{e^{\frac{\left(\theta_{5} \theta_{8}\right)^{2} \theta_{4}}{2}}}{\sqrt{2 \pi}}\left(\theta_{5} \sqrt{\theta_{4}} \theta_{8}\right)^{n} I_{n}\left(s ;-\theta_{8},-\frac{1}{\theta_{5} \sqrt{\theta_{4}}},-\theta_{8} \theta_{5} \sqrt{\theta_{4}}\right)\right), \\
& T_{2, n+1}(s, \Theta)=\frac{\theta_{9}}{\theta_{9}+\theta_{12}}\left(T_{2, n}(s, \Theta)+\theta_{12} \frac{e^{\frac{\left(\theta_{5} \theta_{8}\right)^{2} \theta_{4}}{2}}}{\sqrt{2 \pi}}\left(\theta_{5} \sqrt{\theta_{4}} \theta_{9}\right)^{n} I_{n}\left(s ; \theta_{9}, \frac{1}{\theta_{5} \sqrt{\theta_{4}}},-\theta_{9} \theta_{5} \sqrt{\theta_{4}}\right)\right) \\
& T_{3, n+1}(s, \Theta)=1-T_{2, n+1}(-s, \tilde{\Theta}), \quad T_{4, n+1}(s, \Theta)=1-T_{1, n+1}(-s, \tilde{\Theta}), \\
& T_{1,0}(s, \Theta)=T_{2,0}(s, \Theta)=\theta_{12} \frac{e^{\left(\theta_{5} \theta_{12}\right)^{2} \theta_{4} / 2}}{\sqrt{2 \pi}} I_{0}\left(s ;-\theta_{12},-\frac{1}{\theta_{5} \sqrt{\theta_{4}}},-\theta_{12} \theta_{5} \sqrt{\theta_{4}}\right) \\
& T_{1,1}(s, \Theta)=\frac{1}{\frac{1}{\theta_{8}}-\frac{1}{\theta_{12}}}\left(\frac{e^{\frac{\left(\theta_{5} \theta_{8}\right)^{2} \theta_{4}}{2}}}{\sqrt{2 \pi}} I_{0}\left(s ;-\theta_{8}, \frac{-1}{\theta_{5} \sqrt{\theta_{4}}},-\theta_{8} \theta_{5} \sqrt{\theta_{4}}\right)-\frac{e^{\frac{\left(\theta_{5} \theta_{12}\right)^{2} \theta_{4}}{2}}}{\sqrt{2 \pi}} I_{0}\left(s ;-\theta_{12}, \frac{-1}{\theta_{5} \sqrt{\theta_{4}}},-\theta_{12} \theta_{5} \sqrt{\theta_{4}}\right)\right), \\
& T_{2,1}(s, \Theta)=\frac{1}{\frac{1}{\theta_{9}}-\frac{1}{\theta_{12}}}\left(\frac{e^{\frac{\left(\theta_{5} \theta_{9}\right)^{2} \theta_{4}}{2}}}{\sqrt{2 \pi}} I_{0}\left(s ;-\theta_{9}, \frac{-1}{\theta_{5} \sqrt{\theta_{4}}},-\theta_{9} \theta_{5} \sqrt{\theta_{4}}\right)-\frac{e^{\frac{\left(\theta_{5} \theta_{12}\right)^{2} \theta_{4}}{2}}}{\sqrt{2 \pi}} I_{0}\left(s ;-\theta_{12}, \frac{-1}{\theta_{5} \sqrt{\theta_{4}}},-\theta_{12} \theta_{5} \sqrt{\theta_{4}}\right)\right), \\
& P_{n, m}=\sum_{i=m}^{n-1}\left(\begin{array}{c}
n-m-1 \\
i-m
\end{array}\right)\left(\begin{array}{c}
n \\
i
\end{array}\right)\left(\frac{\theta_{8}}{\theta_{8}+\theta_{9}}\right)^{i-m}\left(\frac{\theta_{9}}{\theta_{8}+\theta_{9}}\right)^{n-i} \theta_{7}^{i}\left(1-\theta_{7}\right)^{n-i}, \\
& Q_{n, m}=\sum_{i=m}^{n-1}\left(\begin{array}{c}
n-m-1 \\
i-m
\end{array}\right)\left(\begin{array}{c}
n \\
i
\end{array}\right)\left(\frac{\theta_{8}}{\theta_{8}+\theta_{9}}\right)^{n-i}\left(\frac{\theta_{9}}{\theta_{8}+\theta_{9}}\right)^{i-m} \theta_{7}^{n-i}\left(1-\theta_{7}\right)^{i}, \\
& P_{n, n}=\theta_{7}^{n}, Q_{n, n}=\left(1-\theta_{7}\right)^{n}, P_{0,0}=1, Q_{0,0}=0, \\
& H h_{n}(x)=\frac{1}{n}\left(H h_{n-2}(x)-x H h_{n-1}(x)\right), H h_{0}(x)=\int_{-\infty}^{-x} e^{-t^{2} / 2} d t, I_{n}(k ; \alpha, \beta, \delta)=-\frac{e^{\alpha k}}{\alpha} H h_{n}(\beta k-\delta)+\frac{\beta}{\alpha} I_{n-1}, \\
& I_{-1}(k ; \alpha, \beta, \delta)=\frac{\sqrt{2 \pi}}{\beta} e^{\frac{\alpha \delta}{\beta}+\frac{\alpha^{2}}{2 \beta^{2}}} \begin{cases}\Phi\left(-\beta k+\delta+\frac{\alpha}{\beta}\right) & \text { if } \beta>0 \alpha \neq 0, \\
-\Phi\left(\beta k-\delta-\frac{\alpha}{\beta}\right) & \text { if } \beta<0 \alpha<0 .\end{cases} \\
& \Upsilon(\Theta)-\sum_{n=0}^{M} \frac{\left(\theta_{6} \theta_{4}\right)^{n} e^{-\theta_{6} \theta_{4}}}{n !} Z_{n}(\Theta) \leq 2 \sum_{n=M+1}^{\infty} \frac{\left(\theta_{6} \theta_{4}\right)^{n} e^{-\theta_{6} \theta_{4}}}{n !}=2-2 \sum_{n=0}^{M} \frac{\left(\theta_{6} \theta_{4}\right)^{n} e^{-\theta_{6} \theta_{4}}}{n !} \equiv \epsilon\left(\theta_{6} \theta_{4}, M\right) .
\end{aligned}
$$

\footnotetext{
${ }^{4}$ This can be verified directly from the proof of Proposition 3.1 presented in A.2
} 
Therefore, if we truncate the summations in (3.2) at the $M$ th term, the upper bound for the truncation error is given by

$$
e^{-\alpha} S \epsilon((m+1) \kappa T, M)+e^{-r T} K \epsilon(\kappa T, M) .
$$

This can be computed quickly and used to limit the error to the a priori desired decimal place. For example, take $S=K=100, T=1$ and an error tolerance of 0.01 . Then, with $\kappa \approx 100$, and $m, \alpha<0.001$, we obtain $M=143$.

\section{A.2 Proof of Propositions 3.1}

In order to price the European call option, we need to evaluate the terms $\mathbb{Q}\left\{S_{T}>K\right\}$ and $\mathbb{E}\left\{e^{S_{T}} 1_{\left\{S_{T}>K\right\}}\right\}$. We first state some useful facts (see Kou (2002) for proofs).

Lemma A.1. Define two i.i.d. exponential r.v.'s, namely, $J_{i}^{+} \sim \operatorname{Exp}\left(\lambda_{1}\right), J_{i}^{-} \sim \operatorname{Exp}\left(\lambda_{2}\right)$. For every $n \geq 1$, we have

$$
\sum_{i=1}^{n} J_{i} \stackrel{d}{=} \begin{cases}\sum_{i=1}^{m} & J_{i}^{+}, \text {w.p. } P_{n, m} \\ \sum_{i=1}^{m} & J_{i}^{-}, \text {w.p. } Q_{n, m}\end{cases}
$$

where

$$
\begin{aligned}
P_{n, m} & =\sum_{i=m}^{n-1}\left(\begin{array}{c}
n-m-1 \\
i-m
\end{array}\right)\left(\begin{array}{c}
n \\
i
\end{array}\right)\left(\frac{\lambda_{1}}{\lambda_{1}+\lambda_{2}}\right)^{i-m}\left(\frac{\lambda_{2}}{\lambda_{1}+\lambda_{2}}\right)^{n-i} p^{i} q^{n-i}, \\
Q_{n, m} & =\sum_{i=m}^{n-1}\left(\begin{array}{c}
n-m-1 \\
i-m
\end{array}\right)\left(\begin{array}{c}
n \\
i
\end{array}\right)\left(\frac{\lambda_{1}}{\lambda_{1}+\lambda_{2}}\right)^{n-i}\left(\frac{\lambda_{2}}{\lambda_{1}+\lambda_{2}}\right)^{i-m} p^{n-i} q^{i}, \\
P_{n, n} & =p^{n}, Q_{n, n}=q^{n}, P_{0,0}=1, Q_{0,0}=0, q=1-p .
\end{aligned}
$$

Next, for every $n \geq 0$, we define the functions 5

$$
\begin{gathered}
H h_{n}(x)=\int_{x}^{\infty} H h_{n-1}(y) d y=\frac{1}{n !} \int_{x}^{\infty}(t-x)^{n} e^{-\frac{t^{2}}{2}} d t \\
H h_{-1}(x)=e^{-x^{2} / 2}, H h_{0}(x)=\int_{x}^{\infty} e^{-\frac{t^{2}}{2}} d t=\sqrt{2 \pi} \Phi(-x),
\end{gathered}
$$

where $\Phi(x)$ denotes the standard normal c.d.f. In addition, for $n \geq 0$, define the integral

$$
I_{n}(k, \alpha, \beta, \delta)=\int_{k}^{\infty} e^{\alpha x} H h_{n}(\beta x-\delta) d x .
$$

Lemma A.2. (i) If $\beta>0$ and $\alpha \neq 0$, then for all $n \geq-1$, we have

$$
I_{n}(k, \alpha, \beta, \delta)=\frac{-e^{\alpha k}}{\alpha} \sum_{i=0}^{n}\left(\frac{\beta}{\alpha}\right)^{n-i} H h_{i}(\beta k-\delta)+\left(\frac{\beta}{\alpha}\right)^{n+1} \frac{\sqrt{2 \pi}}{\beta} e^{\frac{\alpha \delta}{\beta}+\frac{\alpha^{2}}{2 \beta^{2}}} \Phi\left(-\beta k+\delta+\frac{\alpha}{\beta}\right) .
$$

${ }^{5}$ The $H h$ functions can be calculated using either of the following facts:

$$
\begin{aligned}
H h_{n} & =2^{-\frac{n}{2}} \sqrt{\pi} e^{-\frac{x^{2}}{2}}\left(\frac{{ }_{1} F_{1}\left(\frac{n+1}{2}, \frac{1}{2}, \frac{x^{2}}{2}\right)}{\sqrt{2} \Gamma\left(1+\frac{n}{2}\right)}-x \frac{{ }_{1} F_{1}\left(\frac{n}{2}+1, \frac{3}{2}, \frac{x^{2}}{2}\right)}{\Gamma\left(\frac{n+1}{2}\right)}\right), \\
n H h_{n}(x) & =H h_{n-2}(x)-x H h_{n-1}(x), n \geq 1,
\end{aligned}
$$

where ${ }_{1} F_{1}$ denotes the confluent hypergeometric function and $\Gamma$ is the gamma function. 
(ii) If $\beta<0$ and $\alpha<0$, then for all $n \geq-1$, we have

$$
I_{n}(k, \alpha, \beta, \delta)=\frac{-e^{\alpha k}}{\alpha} \sum_{i=0}^{n}\left(\frac{\beta}{\alpha}\right)^{n-i} H h_{i}(\beta k-\delta)-\left(\frac{\beta}{\alpha}\right)^{n+1} \frac{\sqrt{2 \pi}}{\beta} e^{\frac{\alpha \delta}{\beta}+\frac{\alpha^{2}}{2 \beta^{2}}} \Phi\left(\beta k-\delta-\frac{\alpha}{\beta}\right) .
$$

In particular,

$$
I_{-1}(k ; \alpha, \beta, \delta)=\frac{\sqrt{2 \pi}}{\beta} e^{\frac{\alpha \delta}{\beta}+\frac{\alpha^{2}}{2 \beta^{2}}} \begin{cases}\Phi\left(-\beta k+\delta+\frac{\alpha}{\beta}\right) & \text { if } \beta>0, \alpha \neq 0, \\ -\Phi\left(\beta k-\delta-\frac{\alpha}{\beta}\right) & \text { if } \beta<0, \alpha<0 .\end{cases}
$$

In addition, under either assumption (i) or (ii) over the parameters $\alpha$ and $\beta$ given above, the functions $\left(I_{n}\right)$ satisfy the recursive relation

$$
I_{n}(k ; \alpha, \beta, \delta)=-\frac{e^{\alpha k}}{\alpha} H h_{n}(\beta k-\delta)+\frac{\beta}{\alpha} I_{n-1}(k ; \alpha, \beta, \delta) .
$$

In our extended Kou model, we need to understand the distribution of the sum of double exponentially distributed randomly timed and EA jumps. Hence, we consider the associated p.d.f.'s.

Lemma A.3. Let $Z_{e}^{+}, Z_{e}^{-}$be i.i.d. exponential r.v.'s, $Z_{e}^{+}, \sim \operatorname{Exp}\left(\eta_{1}\right), Z_{e}^{-}, \sim \operatorname{Exp}\left(\eta_{2}\right)$. In addition, let $J_{i}^{+}, J_{i}^{-}, Z_{e}^{+}, Z_{e}^{-}$be independent. Then, we have

$$
\begin{aligned}
& f_{\sum_{1}^{n} J_{i}^{+}+Z_{e}^{+}}(t)=\left(\frac{1}{\frac{1}{\lambda_{1}}-\frac{1}{\eta_{1}}}\right)^{n}\left(A_{n}(t) e^{-\lambda_{1} t}+B_{n}(t) e^{-\eta_{1} t}\right), \quad t>0, \lambda_{1} \neq \eta_{1}, \\
& f_{-\sum_{1}^{n} J_{i}^{-}+Z_{e}^{+}}(t)=\left(\frac{1}{\frac{1}{\lambda_{2}}+\frac{1}{\eta_{1}}}\right)^{n} C_{n}(t) e^{\lambda_{2} \min (0, t)-\eta_{1} \max (0, t)}, \quad t \in \mathbb{R}, \lambda_{2} \neq \eta_{1},
\end{aligned}
$$

where $f_{Y}$ denotes the p.d.f. of $Y$, and

$$
\begin{gathered}
B_{n}(t)=-\eta_{1}^{-(n-1)}, A_{n}(t)=1+\sum_{i}^{n-1} \eta_{1}^{-(i-1)}\left(\frac{1}{\lambda_{1}}-\frac{1}{\eta_{1}}\right)^{n-i} \lambda_{1}^{n-i} \frac{(t)^{n-i}}{(n-i) !}, \quad t>0 \\
C_{n}(t)=\frac{C_{n-1}(t)}{\lambda_{2}}+\lambda_{2}^{n-1} \frac{(\max (0, t)-t)^{n-1}}{(n-1) !}, \quad t \in \mathbb{R} .
\end{gathered}
$$

In addition, they satisfy the following recursive relations:

$$
\begin{gathered}
f_{\sum_{1}^{n+1} J_{i}^{+}+Z_{e}^{+}}(t)=\frac{\lambda_{1}}{\left(\lambda_{1}-\eta_{1}\right)}\left(f_{\sum_{1}^{n} J_{i}^{+}+Z_{e}^{+}}(t)-\lambda_{1}^{n} \frac{(t)^{n}}{n !} \eta_{1} e^{-\lambda_{1} t}\right), \\
f_{-\sum_{1}^{n+1} J_{i}^{-}+Z_{e}^{+}}(t)=\frac{\lambda_{2}}{\lambda_{2}+\eta_{1}}\left(f_{-\sum_{1}^{n} J_{i}^{-}+Z_{e}^{+}}(t)+\lambda_{2}^{n} \frac{(\max (0, t)-t)^{n}}{n !} \eta_{1} e^{\lambda_{2} t} e^{-\left(\lambda_{2}+\eta_{1}\right) \max (0, t)}\right) .
\end{gathered}
$$

Proof. We first notice that the positive r.v., $J_{i}^{+}+Z_{e}^{+}$, has the p.d.f.

$$
\begin{aligned}
\int_{0}^{t} \lambda_{1} e^{-\lambda_{1}(t-x)} \eta_{1} e^{-\eta_{1} x} d x & =\frac{1}{\frac{1}{\lambda_{1}}-\frac{1}{\eta_{1}}}\left(e^{-\lambda_{1} t}-e^{-\eta_{1} t}\right) \\
& \equiv\left(\frac{1}{\frac{1}{\lambda_{1}}-\frac{1}{\eta_{1}}}\right)\left(A_{1}(t) e^{-\lambda_{1} t}+B_{1}(t) e^{-\eta_{1} t}\right), \quad t>0, \lambda_{1} \neq \eta_{1} .
\end{aligned}
$$


Now suppose for a fixed $n \geq 1, f_{\sum_{1}^{n} J_{i}^{+}+Z_{e}^{+}}(t)=\left(\frac{1}{\frac{1}{\lambda_{1}}-\frac{1}{\eta_{1}}}\right)^{n}\left(A_{n}(t) e^{-\lambda_{1} t}+B_{n}(t) e^{-\eta_{1} t}\right)$, we obtain

$$
\begin{aligned}
f_{\sum_{1}^{n} J_{i}^{+}+Z_{e}^{+}}(t) & =\int_{0}^{t} \lambda_{1}^{n} \frac{(t-x)^{n-1}}{(n-1) !} e^{-\lambda_{1}(t-x)} \eta_{1} e^{-\eta_{1} x} d x \\
& =\lambda_{1}^{n} \frac{(t)^{n}}{n !} \eta_{1} e^{-\lambda_{1} t}+\frac{\left(\lambda_{1}-\eta_{1}\right)}{\lambda_{1}} f_{\sum_{1}^{n+1} J_{i}^{+}+Z_{e}^{+}}(t), \\
\Longrightarrow f_{\sum_{1}^{n+1} J_{i}^{+}+Z_{e}^{+}}(t) & =\frac{\lambda_{1}}{\left(\lambda_{1}-\eta_{1}\right)}\left(f_{\sum_{1}^{n} J_{i}^{+}+Z_{e}^{+}}(t)-\lambda_{1}^{n} \frac{(t)^{n}}{n !} \eta_{1} e^{-\lambda_{1} t}\right) \\
& =\left(\frac{1}{\frac{1}{\lambda_{1}}-\frac{1}{\eta_{1}}}\right)^{n+1}\left(A_{n+1}(t) e^{-\lambda_{1} t}+B_{n+1}(t) e^{-\eta_{1} t}\right),
\end{aligned}
$$

where the coefficients satisfy

$$
\begin{aligned}
& B_{n+1}=\frac{1}{\eta_{1}} B_{n}, \quad A_{n+1}=\frac{A_{n}}{\eta_{1}}-C_{n+1}, \quad C_{n+1}=\left(\frac{1}{\lambda_{1}}-\frac{1}{\eta_{1}}\right)^{n} \lambda_{1}^{n} \frac{(t)^{n}}{n !} \\
& B_{n}=-\eta_{1}^{-(n-1)}, \quad A_{n}=1+\sum_{i}^{n-1} \eta_{1}^{-(i-1)}\left(\frac{1}{\lambda_{1}}-\frac{1}{\eta_{1}}\right)^{n-i} \lambda_{1}^{n-i} \frac{(t)^{n-i}}{(n-i) !}
\end{aligned}
$$

and this yields (A.2). Next, we note that the real-valued r.v. $-J_{i}^{-}+Z_{e}^{+}$has the p.d.f.

$$
\int_{\max (0, t)}^{\infty} \lambda_{2} e^{\lambda_{2}(t-x)} \eta_{1} e^{-\eta_{1} x} d x=\frac{e^{\lambda_{2} \min (0, t)-\eta_{1} \max (0, t)}}{\frac{1}{\lambda_{2}}+\frac{1}{\eta_{1}}}, \quad t \in \mathbb{R}, \lambda_{2} \neq \eta_{1} .
$$

For a fixed $n \geq 1$, assume that $f_{-\sum_{1}^{n} J_{i}^{-}+Z_{e}^{+}}(t)=\left(\frac{1}{\frac{1}{\lambda_{2}}+\frac{1}{\eta_{1}}}\right)^{n} C_{n}(t) e^{\lambda_{2} \min (0, t)-\eta_{1} \max (0, t)}$, then we get

$$
\begin{aligned}
f_{-\sum_{1}^{n} J_{i}^{-}+Z_{e}^{+}}(t) & =\int_{\max (0, t)}^{\infty} \lambda_{2}^{n} \frac{(-t+x)^{n-1}}{(n-1) !} e^{\lambda_{2}(t-x)} \eta_{1} e^{-\eta_{1} x} d x \\
& =-\lambda_{2}^{n} \frac{(\max (0, t)-t)^{n}}{n !} \eta_{1} e^{\lambda_{2} t} e^{-\left(\lambda_{1}+\eta_{1}\right) \max (0, t)}+\frac{\left(\lambda_{2}+\eta_{1}\right)}{\lambda_{2}} f_{-\sum_{1}^{n+1} J_{i}^{-}+Z_{e}^{+}}(t), \\
\Longrightarrow f_{-\sum_{1}^{n+1} J_{i}^{-}+Z_{e}^{+}}(t) & =\frac{\lambda_{2}}{\lambda_{2}+\eta_{1}}\left(f_{-\sum_{1}^{n} J_{i}^{-}+Z_{e}^{+}}(t)+\lambda_{2}^{n} \frac{(\max (0, t)-t)^{n}}{n !} \eta_{1} e^{\lambda_{2} t} e^{-\left(\lambda_{1}+\eta_{1}\right) \max (0, t)}\right) \\
& \equiv\left(\frac{1}{\frac{1}{\lambda_{2}}+\frac{1}{\eta_{1}}}\right)^{n+1} C_{n+1}(t) e^{\lambda_{2} \min (0, t)-\eta_{1} \max (0, t)} .
\end{aligned}
$$

Matching terms yields $C_{n+1}(t)=\frac{C_{n}(t)}{\lambda_{2}}+\lambda_{2}^{n} \frac{(\max (0, t)-t)^{n}}{n !}$.

We now calculate the distribution of the sum of a normal r.v. and double-exponentials.

Proposition A.4. Let $W \sim N\left(0, \sigma^{2}\right)$. Then, we have the p.d.f.'s:

$$
\begin{aligned}
f_{W+\sum_{i=1}^{n+1} J_{i}^{+}+Z_{e}^{+}}(t) & =\left(\frac{\lambda_{1}}{\lambda_{1}-\eta_{1}}\right)^{n} \eta_{1} \frac{e^{\frac{\left(\sigma \eta_{1}\right)^{2}}{2}}}{\sqrt{2 \pi}} e^{-\eta_{1} t} H h_{0}\left(-\frac{t}{\sigma}+\sigma \eta_{1}\right)+ \\
& -\sum_{i=1}^{n}\left(\frac{\lambda_{1}}{\lambda_{1}-\eta_{1}}\right)^{n-i+1} \eta_{1} \frac{e^{\frac{\left(\sigma \lambda_{1}\right)^{2}}{2}}}{\sqrt{2 \pi}}\left(\sigma^{i} \lambda_{1}^{i}\right) e^{-\lambda_{1} t} H h_{i}\left(\frac{-t}{\sigma}+\sigma \lambda_{1}\right), t>0, \lambda_{1} \neq \eta_{1},
\end{aligned}
$$




$$
\begin{aligned}
f_{W-\sum_{i=1}^{n+1} J_{i}^{-}+Z_{e}^{+}}(t) & =\left(\frac{\lambda_{2}}{\lambda_{2}+\eta_{1}}\right)^{n} \eta_{1} \frac{e^{\frac{\left(\sigma \eta_{1}\right)^{2}}{2}}}{\sqrt{2 \pi}} e^{-\eta_{1} t} H h_{0}\left(-\frac{t}{\sigma}+\sigma \eta_{1}\right)+ \\
& +\sum_{i=1}^{n}\left(\frac{\lambda_{2}}{\lambda_{2}+\eta_{1}}\right)^{n-i+1} \eta_{1} \frac{e^{\frac{\left(\sigma \lambda_{2}\right)^{2}}{2}}}{\sqrt{2 \pi}}\left(\sigma^{i} \lambda_{2}^{i}\right) e^{\lambda_{2} t} H h_{i}\left(\frac{t}{\sigma}+\sigma \lambda_{2}\right), t \in \mathbb{R}, \lambda_{2} \neq \eta_{1} .
\end{aligned}
$$

Moreover, they admit the recursive relations:

$$
\begin{aligned}
f_{W+\sum_{i=1}^{n+1} J_{i}^{+}+Z_{e}^{+}}(t) & =\frac{\lambda_{1}}{\lambda_{1}-\eta_{1}}\left(f_{W+\sum_{i=1}^{n} J_{i}^{+}+Z_{e}^{+}}(t)-\eta_{1} \frac{e^{\frac{\left(\sigma \lambda_{1}\right)^{2}}{2}}}{\sqrt{2 \pi}}\left(\sigma^{n} \lambda_{1}^{n}\right) e^{-\lambda_{1} t} H h_{n}\left(\frac{-t}{\sigma}+\sigma \lambda_{1}\right)\right), \\
f_{W-\sum_{i=1}^{n+1} J_{i}^{-}+Z_{e}^{+}}(t) & =\frac{\lambda_{2}}{\lambda_{2}+\eta_{1}}\left(f_{W-\sum_{i=1}^{n+1} J_{i}^{-}+Z_{e}^{+}}(t)+\eta_{1} \frac{e^{\frac{\left(\sigma \lambda_{2}\right)^{2}}{2}}}{\sqrt{2 \pi}}\left(\sigma^{n} \lambda_{2}^{n}\right) e^{\lambda_{2} t} H h_{n}\left(\frac{t}{\sigma}+\sigma \lambda_{2}\right)\right) .
\end{aligned}
$$

Proof. We start with the p.d.f. for $W+Z_{e}^{+}$:

$$
f_{W+Z_{e}^{+}}(t)=\eta_{1} \frac{e^{\frac{\left(\sigma \eta_{1}\right)^{2}}{2}}}{\sqrt{2 \pi}} e^{-\eta_{1} t} H h_{0}\left(-\frac{t}{\sigma}+\sigma \eta_{1}\right) .
$$

Using (A.4), we also write

$$
\begin{aligned}
f_{W+\sum_{i=1}^{n+1} J_{i}^{+}+Z_{e}^{+}}(t) & =\int_{-\infty}^{t} f_{\sum_{1}^{n+1} J_{i}^{+}+Z_{e}^{+}}(t-x) \frac{e^{-\frac{x^{2}}{2 \sigma^{2}}}}{\sqrt{2 \pi \sigma^{2}}} d x \\
& =\frac{\lambda_{1}}{\lambda_{1}-\eta_{1}}\left(f_{W+\sum_{i=1}^{n} J_{i}^{+}+Z_{e}^{+}}(t)-\eta_{1} \frac{e^{\frac{\left(\sigma \lambda_{1}\right)^{2}}{2}}}{\sqrt{2 \pi}}\left(\sigma^{n} \lambda_{1}^{n}\right) e^{-\lambda_{1} t} H h_{n}\left(\frac{-t}{\sigma}+\sigma \lambda_{1}\right)\right),
\end{aligned}
$$

which leads directly to

$$
\begin{aligned}
f_{W+\sum_{i=1}^{n+1} J_{i}^{+}+Z_{e}^{+}}(t)= & \left(\frac{\lambda_{1}}{\lambda_{1}-\eta_{1}}\right)^{n} \eta_{1} \frac{e^{\frac{\left(\sigma \eta_{1}\right)^{2}}{2}}}{\sqrt{2 \pi}} e^{-\eta_{1} t} H h_{0}\left(-\frac{t}{\sigma}+\sigma \eta_{1}\right)+ \\
& -\sum_{i=1}^{n}\left(\frac{\lambda_{1}}{\lambda_{1}-\eta_{1}}\right)^{n-i+1} \eta_{1} \frac{e^{\frac{\left(\sigma \lambda_{1}\right)^{2}}{2}}}{\sqrt{2 \pi}}\left(\sigma^{i} \lambda_{1}^{i}\right) e^{-\lambda_{1} t} H h_{i}\left(\frac{-t}{\sigma}+\sigma \lambda_{1}\right) .
\end{aligned}
$$

To prove (A.7), we apply (A.5) to get the recursive expression

$$
\begin{aligned}
f_{W-\sum_{i=1}^{n+1} J_{i}^{-}+Z_{e}^{+}}(t) & =\int_{\mathbb{R}} f_{-\sum_{i=1}^{n+1} J_{i}^{-}+Z_{e}^{+}}(t-x) \frac{e^{-\frac{x^{2}}{2 \sigma^{2}}}}{\sqrt{2 \pi \sigma^{2}}} d x \\
& =\frac{\lambda_{2}}{\lambda_{2}+\eta_{1}}\left(f_{W-\sum_{i=1}^{n+1} J_{i}^{-}+Z_{e}^{+}}(t)+\eta_{1} \frac{e^{\frac{\left(\sigma \lambda_{2}\right)^{2}}{2}}}{\sqrt{2 \pi}}\left(\sigma^{n} \lambda_{2}^{n}\right) e^{\lambda_{2} t} H h_{n}\left(\frac{t}{\sigma}+\sigma \lambda_{2}\right)\right),
\end{aligned}
$$


which can be written explicitly as

$$
\begin{aligned}
f_{W-\sum_{i=1}^{n+1} J_{i}^{-}+Z_{e}^{+}}(t)= & \left(\frac{\lambda_{2}}{\lambda_{2}+\eta_{1}}\right)^{n} \eta_{1} \frac{e^{\frac{\left(\sigma \eta_{1}\right)^{2}}{2}}}{\sqrt{2 \pi}} e^{-\eta_{1} t} H h_{0}\left(-\frac{t}{\sigma}+\sigma \eta_{1}\right)+ \\
& +\sum_{i=1}^{n}\left(\frac{\lambda_{2}}{\lambda_{2}+\eta_{1}}\right)^{n-i+1} \eta_{1} \frac{e^{\frac{\left(\sigma \lambda_{2}\right)^{2}}{2}}}{\sqrt{2 \pi}}\left(\sigma^{i} \lambda_{2}^{i}\right) e^{\lambda_{2} t} H h_{i}\left(\frac{t}{\sigma}+\sigma \lambda_{2}\right) .
\end{aligned}
$$

We can now calculate the tail probabilities that will allow us to price the call option.

Proposition A.5. Let $F_{X}(z) \equiv \mathbb{Q}\{X \leq z\}$. Then,

$$
\begin{aligned}
F_{W+\sum_{i=1}^{n+1} J_{i}^{+}+Z_{e}^{+}}(z) & =\left(\frac{\lambda_{1}}{\lambda_{1}-\eta_{1}}\right)^{n} \eta_{1} \frac{e^{\frac{\left(\sigma \eta_{1}\right)^{2}}{2}}}{\sqrt{2 \pi}} I_{0}\left(z ;-\eta_{1},-\frac{1}{\sigma},-\eta_{1} \sigma\right)+ \\
& -\sum_{i=1}^{n}\left(\frac{\lambda_{1}}{\lambda_{1}-\eta_{1}}\right)^{n-i+1} \eta_{1} \frac{e^{\frac{\left(\sigma \lambda_{1}\right)^{2}}{2}}}{\sqrt{2 \pi}}\left(\sigma^{i} \lambda_{1}^{i}\right) I_{i}\left(z ;-\lambda_{1},-\frac{1}{\sigma},-\lambda_{1} \sigma\right), \quad z>0, \lambda_{1} \neq \eta_{1}, \\
F_{W-\sum_{i=1}^{n+1} J_{i}^{-}+Z_{e}^{+}}(z) & =\left(\frac{\lambda_{2}}{\lambda_{2}+\eta_{1}}\right)^{n} \eta_{1} \frac{e^{\frac{\left(\sigma \eta_{1}\right)^{2}}{2}}}{\sqrt{2 \pi}} I_{0}\left(z,-\eta_{1},-\frac{1}{\sigma},-\sigma \eta_{1}\right)+ \\
& +\sum_{i=1}^{n}\left(\frac{\lambda_{2}}{\lambda_{2}+\eta_{1}}\right)^{n-i+1} \eta_{1} \frac{e^{\frac{\left(\sigma \lambda_{2}\right)^{2}}{2}}}{\sqrt{2 \pi}}\left(\sigma^{i} \lambda_{2}^{i}\right) I_{n}\left(z, \lambda_{2}, \frac{1}{\sigma},-\sigma \lambda_{2}\right), \quad z \in \mathbb{R}, \lambda_{2} \neq \eta_{1},
\end{aligned}
$$

In addition, these c.d.f.'s admit the following recursive relations:

$$
\begin{aligned}
& F_{W+\sum_{i=1}^{n+1} J_{i}^{+}+Z_{e}^{+}}(z)=\frac{\lambda_{1}}{\left(\lambda_{1}-\eta_{1}\right)}\left(F_{W+\sum_{i=1}^{n} J_{i}^{+}+Z_{e}^{+}}(z)-\eta_{1} \frac{e^{\frac{\left(\sigma \lambda_{1}\right)^{2}}{2}}}{\sqrt{2 \pi}}\left(\sigma^{n} \lambda_{1}^{n}\right) I_{n}\left(z ;-\lambda_{1},-\frac{1}{\sigma},-\lambda_{1} \sigma\right)\right), \\
& F_{W-\sum_{i=1}^{n+1} J_{i}^{-}+Z_{e}^{+}}(z)=\frac{\lambda_{2}}{\lambda_{2}+\eta_{1}}\left(F_{W-\sum_{i=1}^{n+1} J_{i}^{-}+Z_{e}^{+}}(z)+\eta_{1} \frac{e^{\frac{\left(\sigma \lambda_{2}\right)^{2}}{2}}}{\sqrt{2 \pi}}\left(\sigma^{n} \lambda_{2}^{n}\right) I_{n}\left(z, \lambda_{2}, \frac{1}{\sigma},-\sigma \lambda_{2}\right)\right) .
\end{aligned}
$$

Proof. The above expressions can all be derived by integrating the corresponding densities given in (A.6), (A.7), (A.8), (A.9) and taking into account the definitions of the $H h$ and $I$ functions.

We remark that although we did not provide the tail probability formulas $F_{\sigma W_{T}+\sum_{i=1}^{m} \hat{J}_{i}^{+}-\hat{Z}_{e}^{-}}$and $F_{\sigma W_{T}-\sum_{i=1}^{m} \hat{J}_{i}^{-}-\hat{Z}_{e}^{-}}$we point out that they can be derived by symmetry. For example, we have

$$
F_{\sigma W_{T}+\sum_{i=1}^{m} \hat{J}_{i}^{+}-\hat{Z}_{e}^{-}}(s)=1-F_{\sigma W_{T}-\sum_{i=1}^{m} \hat{J}_{i}^{+}+\hat{Z}_{e}^{-}(-s) .}
$$

In fact, these c.d.f.'s appear in the pricing formula as the functions $T_{i, j}$ 's, defined in Section A.1, We now give the formula for the call option price. Recall the call price can be written as

$$
C(S)=S \mathbb{E}\left\{e^{\left(-\frac{\sigma^{2}}{2}-\frac{\alpha}{T}-\kappa \zeta\right) T+\sigma W_{T}+\sum_{i=1}^{N_{T}} J_{i}+Z_{e}} \mathbf{1}_{\left\{S_{T}>K\right\}} \mid S_{0}=S\right\}-e^{-r T} K \mathbb{Q}\left\{S_{T}>K \mid S_{0}=S\right\} .
$$


Using the results above, we can write

$$
\begin{aligned}
& \mathbb{Q}\left\{S_{T}>K \mid S_{0}=S\right\} \\
= & \sum_{n=1}^{\infty} \mathbb{Q}\left\{N_{T}=n\right\} \mathbb{Q}\left\{\sigma W_{T}+\sum_{i}^{n} J_{i}+Z_{e}>\log \left(\frac{K}{S}\right)-\left(r-\frac{\sigma^{2}}{2}-\frac{\alpha}{T}-\kappa \zeta\right) T\right\} \\
= & \sum_{n=1}^{\infty} \frac{\kappa^{n} e^{-\kappa}}{n !}\left[u \sum_{m=1}^{n}\left(P_{n, m} \mathbb{Q}\left\{\sigma W_{T}+\sum_{i}^{m} J_{i}^{+}+Z_{e}^{+}>k\right\}+Q_{n, m} \mathbb{Q}\left\{\sigma W_{T}-\sum_{i}^{m} J_{i}^{-}+Z_{e}^{+}>k\right\}\right)+\right. \\
& \left.+w \sum_{m=1}^{n}\left(P_{n, m} \mathbb{Q}\left\{\sigma W_{T}+\sum_{i}^{m} J_{i}^{+}-Z_{e}^{-}>k\right\}+Q_{n, m} \mathbb{Q}\left\{\sigma W_{T}-\sum_{i}^{m} J_{i}^{-}-Z_{e}^{-}>k\right\}\right)\right] \\
= & \sum_{n=1}^{\infty} \frac{\kappa^{n} e^{-\kappa}}{n !}\left[u \sum_{m=1}^{n}\left(P_{n, m} T_{1, n}(k, \Theta)+Q_{n, m} T_{2, n}(k, \Theta)\right)+w \sum_{m=1}^{n}\left(P_{n, m} T_{3, n}(k, \Theta)+Q_{n, m} T_{4, n}(k, \Theta)\right)\right]
\end{aligned}
$$

where $k \equiv \log \left(\frac{K}{S}\right)-\left(r-\frac{\sigma^{2}}{2}-\kappa \zeta\right) T-\alpha$.

It remains to compute

$$
\begin{aligned}
& \mathbb{E}\left\{e^{\sigma W_{T}+\sum_{i=1}^{N_{T}} J_{i}+Z_{e}} \mathbf{1}_{\left\{S_{T}>K\right\}} \mid S_{0}=S\right\} \\
& \quad=\sum_{n=1}^{\infty} \frac{(\kappa T)^{n} e^{-\kappa T}}{n !}\left[u \sum_{m=1}^{n} P_{n, m} \mathbb{E}\left\{e^{\sigma W_{T}+\sum_{i=1}^{m} J_{i}^{+}+Z_{e}^{+}} \mathbf{1}_{\left\{\sigma W_{T}+\sum_{i=1}^{m} J_{i}^{+}+Z_{e}^{+}>k\right\}}\right\}+\right. \\
& +u \sum_{k=1}^{n} Q_{n, k} \mathbb{E}\left\{e^{\sigma W_{T}-\sum_{i=1}^{m} J_{i}^{-}+Z_{e}^{+}} \mathbf{1}_{\left\{\sigma W_{T}-\sum_{i=1}^{m} J_{i}^{-}+Z_{e}^{+}>k\right\}}\right\}+ \\
& \quad+w \sum_{k=1}^{n} P_{n, k} \mathbb{E}\left\{e^{\sigma W_{T}+\sum_{i=1}^{m} J_{i}^{+}-Z_{e}^{-}} \mathbf{1}_{\left\{\sigma W_{T}+\sum_{i=1}^{m} J_{i}^{+}-Z_{e}^{-}>k\right\}}\right\}+ \\
& \left.\quad+w \sum_{k=1}^{n} Q_{n, k} \mathbb{E}\left\{e^{\sigma W_{T}-\sum_{i=1}^{m} J_{i}^{-}-Z_{e}^{-}} \mathbf{1}_{\left\{\sigma W_{T}-\sum_{i=1}^{m} J_{i}^{-}-Z_{e}^{-}\right\}}\right\}\right]
\end{aligned}
$$

In order to compute the expectation in (A.17), we apply the p.d.f. from Prop. A.4 to write

$$
\begin{aligned}
\mathbb{E} & \left\{e^{\sigma W_{T}+\sum_{i=1}^{m} J_{i}^{+}+Z_{e}^{+}} \mathbf{1}_{\left\{\sigma W_{T}+\sum_{i=1}^{m} J_{i}^{+}+Z_{e}^{+}>k\right\}}\right\}= \\
& =\int_{k}^{\infty} \int_{\mathbb{R}} \frac{e^{t-x} e^{-\frac{(t-x)^{2}}{2 \sigma^{2} T}}}{\sqrt{2 \pi \sigma^{2} T}} \int_{\mathbb{R}} e^{x-y} f_{\sum_{i=1}^{m} J_{i}^{+}}(x-y) e^{y} f_{Z_{e}^{+}}(y) d y d x d t \\
& =\int_{k}^{\infty} \int_{\mathbb{R}} e^{\frac{\sigma^{2} T}{2}} \frac{e^{-\frac{\left(t-x-\sigma^{2} T\right)^{2}}{2 \sigma^{2} T}}}{\sqrt{2 \pi \sigma^{2} T}} \int_{\mathbb{R}}\left(\frac{\lambda_{1}}{\lambda_{1}-1}\right)^{m} f_{\sum_{i=1}^{m} \hat{J}_{i}^{+}}(x-y) \frac{\eta_{1}}{\eta_{1}-1} f_{\hat{Z}_{e}^{+}}(y) d y d x d t \\
& =e^{\frac{\sigma^{2} T}{2}}\left(\frac{\lambda_{1}}{\lambda_{1}-1}\right)^{m} \frac{\eta_{1}}{\eta_{1}-1} \mathbb{Q}\left(\sigma W_{T}+\sum_{i=1}^{m} \hat{J}_{i}^{+}+\hat{Z}_{e}^{+}>k-\sigma^{2} T\right) \\
& =e^{\frac{\sigma^{2} T}{2}}\left(\frac{\lambda_{1}}{\lambda_{1}-1}\right)^{m} \frac{\eta_{1}}{\eta_{1}-1} F_{\sigma W_{T}+\sum_{i=1}^{m} \hat{J}_{i}^{+}+\hat{Z}_{e}^{+}}\left(k-\sigma^{2} T\right),
\end{aligned}
$$

where $\hat{J}_{i}^{+} \sim \operatorname{Exp}\left(\lambda_{1}-1\right)$ and $\hat{Z}_{e}^{+} \sim \operatorname{Exp}\left(\eta_{1}-1\right)$. Expressions analogous to (A.21) can be found for the 
terms (A.18), (A.19) and (A.20). Substituting these expressions and rearranging terms, we get

$$
\begin{aligned}
& \mathbb{E}\left\{e^{\left(-\frac{\sigma^{2}}{2}-\frac{\alpha}{T}-\kappa \zeta\right) T+\sigma W_{T}+\sum_{i=1}^{N} J_{i}+Z_{e}} \mathbf{1}_{\left\{S_{T}>K\right\}} \mid S_{0}=S\right\} \\
& =e^{-\alpha} \sum_{n=1}^{\infty} \frac{(\hat{\kappa} T)^{n} e^{-\hat{\kappa} T}}{n !}\left(\hat{u} \sum_{k=1}^{n}\left(\hat{P}_{n, k} T_{1, n}(k, \Theta)+\hat{Q}_{n, k} T_{2, n}(k, \Theta)\right)+\hat{w} \sum_{k=1}^{n}\left(\hat{P}_{n, k} T_{3, n}(\hat{k}, \Theta)+\hat{Q}_{n, k} T_{4, n}(\hat{k}, \Theta)\right)\right)
\end{aligned}
$$

where $\hat{P}_{n, k}, \hat{Q}_{n, k}$ are calculated as $P_{n, k}, Q_{n, k}$ but with the parameters $\hat{\eta}_{1,2}, \hat{\lambda}_{1,2}$ in place of $\eta_{1,2}$ and $\lambda_{1,2}$. In addition, the Poisson intensity parameter has also been transformed to $\hat{\kappa} \equiv(m+1) \kappa$, with $m=p \frac{\lambda_{1}}{\lambda_{1}-1}+q \frac{\lambda_{2}}{\lambda_{2}+1}-1$. Finally, substituting the expressions for (A.15) and (A.16) into (A.14) concludes the proof.

\section{A.3 Proof of Propositions 4.1 and 4.2}

The first part of Proposition 4.1 follows from Jensen's inequality, namely,

$$
\begin{aligned}
C(t, S) & \geq \mathbb{E}\left\{e^{-r \tau}\left(S e^{r \tau+Z_{e}}-K\right)^{+}\right\} \\
& =\int_{\mathbb{R}^{+}} C_{B S}\left(\tau, S ; \frac{\hat{\sigma}}{\sqrt{\tau}}, K, r\right) \mathbb{G}(d \hat{\sigma}) \geq C_{B S}\left(\tau, S ; \frac{\hat{\sigma}_{\text {min }}}{\sqrt{\tau}}, K, r\right),
\end{aligned}
$$

where $\tau \equiv T-t$. In (A.22), the equality follows from the tower property of the conditional expectation and the last inequality follows from the monotonicity of $C_{B S}$ w.r.t. the volatility parameter $\sigma$. The second part of the proposition is proved in a similar fashion.

To prove Proposition 4.2, we first observe that

$$
C(t, S)=\int_{\mathbb{R}^{+} \times \mathbb{R}^{+}} C_{B S}\left(\tau, S ; \sqrt{\frac{\tilde{\sigma}^{2}+\hat{\sigma}^{2}}{\tau}}, K, r\right) \mathbb{H}(d \tilde{\sigma}) \mathbb{G}(d \hat{\sigma}) .
$$

For an ATM-forward option, i.e. $K=e^{r \tau} S$, we notice that the Black-Scholes price $C_{B S}$ is concave in its volatility parameter $\sigma$. Consequently, by Jensen's inequality, we obtain the upper bound

$$
C(t, S) \leq C_{B S}\left(\tau, S ; \sqrt{\int_{\mathbb{R}^{+}} \frac{\tilde{\sigma}^{2}}{\tau} \mathbb{H}(d \tilde{\sigma})+\int_{\mathbb{R}^{+}} \frac{\hat{\sigma}^{2}}{\tau} \mathbb{G}(d \hat{\sigma})}, K, r\right) .
$$

\section{A.4 Proof of Propositions 4.4 and 4.5}

Propositions 4.4 and 4.5 are applications of the more general results developed in Benaim and Friz (2008). Denote by $M(\omega) \equiv \mathbb{E}\left\{e^{\omega X}\right\}$ the m.g.f. of the r.v. $X$, and with $F$ its c.d.f. As mentioned in Section 4 , if $r^{*} \equiv \inf \{\omega$ s.t. $M(\omega)<\infty\}$ is finite, than it follows that $\lim \sup _{x \rightarrow \infty} \frac{-\log (1-F(x))}{x}=r^{*}$. In turn, if $F$ is well-behaved, the lim sup can be replaced by a lim and the tail asymptotics $\frac{-\log (1-F(x))}{x} \sim r^{*} x$, along with the condition $r^{*}>1$ are sufficient to provide the asymptotics for the implied volatility

$$
\frac{I^{2}(t ; K, T)(T-t)}{\log \left(\frac{K}{S_{t}}\right)} \sim \xi\left(p^{*}\right), \quad \text { as } K \rightarrow \infty ;
$$

see Theorems 9 and 10 of Benaim and Friz (2008) for this result and the associated technical conditions. A symmetric argument holds for the negative tails of $F$ and of the implied volatility. Therefore, given that 
the log stock price under the extended Kou and Heston models admits a m.g.f, it remains to prove that $\frac{-\log (1-F(x))}{x} \sim r^{*} x$. Theorem 7 and Theorem 8 in Benaim and Friz (2008) provide sufficient conditions for models admitting a m.g.f. to ensure that such condition holds.

The m.g.f. $M$ of $X \equiv \log \left(\frac{S_{T}}{S_{t}}\right)$ under the extended Heston model (3.3), given $\sigma_{t}=\sigma$, satisfies 6

$$
\begin{aligned}
& \log M(\omega)=C+\omega D-\frac{\nu \vartheta}{\zeta^{2}}\left(2 \log \left(\frac{1-g e^{-d(T-t)}}{1-g}\right)+d(T-t)\right)+\frac{\nu-\rho \zeta \omega-d}{\zeta^{2}} \frac{1-e^{-d(T-t)}}{1-g e^{-d(T-t)}} \sigma^{2}+\psi_{e}(\omega), \\
& \text { with } g=\frac{\nu-\rho \zeta \omega-d}{\nu-\rho \zeta \omega+d}, \quad d=\sqrt{(\nu-\rho \zeta \omega)^{2}+\zeta^{2}\left(\omega-\omega^{2}\right)}, \quad \psi_{e}(\omega)=\log \left(u \frac{\eta_{1}}{\eta_{1}-\omega}+w \frac{\eta_{2}}{\eta_{2}+\omega}\right)
\end{aligned}
$$

where $C$ and $D$ are constants. Clearly, we have $r^{*}=\min \left\{p, \eta_{1}\right\}$, where $p$ is the smallest positive solution of $1-\left.g e^{-d(T-t)}\right|_{\omega=p}=0$. In turn, the last equation is equivalent to

$$
\nu-\rho \zeta p+\sqrt{(\nu-\rho \zeta p)^{2}+\zeta^{2}\left(p-p^{2}\right)} \operatorname{coth}\left(\frac{(T-t)}{2} \sqrt{(\nu-\rho \zeta p)^{2}+\zeta^{2}\left(p-p^{2}\right)}\right)=0 .
$$

Notice that, when $r^{*}=p$, the dominating term for $\omega \rightarrow p$ is $\frac{\nu-\rho \zeta \omega i-d}{\zeta^{2}} \frac{1-e^{-d(T-t)}}{1-g e^{-d(T-t)}} \sigma^{2}$. Using l'Hopital's rule, it follows that

$$
\frac{p-\omega}{1-g e^{-d(T-t)}} \rightarrow \text { constant, } \quad \text { as } \omega \rightarrow p^{-} .
$$

This means that $\frac{\nu-\rho \zeta \omega-d}{\zeta^{2}} \frac{1-e^{-d(T-t)}}{1-g \rho^{-d(T-t)}} \sigma_{t}^{2}$ is regularly varying of index 1 as a function of $\frac{1}{p-\omega}$ and Criterion II of Theorem 8 in Benaim and Friz (2008) is satisfied. When $r^{*}=\eta_{1}$, we have

$$
M\left(\eta_{1}-z\right) \sim u \eta_{1} z^{-1} \text { as } z \rightarrow 0^{+}
$$

and thus Criterion I of Theorem 7 in Benaim and Friz (2008) is satisfied. A similar argument holds for the negative tail.

Now consider the m.g.f. of $X \equiv \log \left(\frac{S_{T}}{S_{t}}\right)$ under the extended Kou model:

$$
\log M(\omega)=\mu \omega+\frac{\sigma^{2} \omega^{2}}{2}+\kappa\left(p \frac{\lambda_{1}}{\lambda_{1}-\omega}+(1-p) \frac{\lambda_{2}}{\lambda_{2}+\omega}-1\right)+\log \left(u \frac{\eta_{1}}{\eta_{1}-\omega}+(1-u) \frac{\eta_{2}}{\eta_{2}+\omega}\right),
$$

where $\mu$ is a constant. This m.g.f. satisfies the tail asymptotics condition with $r^{*}=\min \left\{\lambda_{1}, \eta_{1}\right\}$, and

$$
\begin{cases}\log M\left(\lambda_{1}-z\right) \sim \kappa p \lambda_{1} z^{-1} \text { as } z \rightarrow 0^{+} & \text {if } \lambda_{1} \leq \eta_{1} \\ M\left(\eta_{1}-z\right) \sim u \eta_{1} z^{-1} \text { as } z \rightarrow 0^{+} & \text {if } \lambda_{1}>\eta_{1}\end{cases}
$$

Hence, $M$ satisfies either Criterion I or II (depending on whether $\lambda_{1}>\eta_{1}$ or not) of Theorems 7 and 8 in Benaim and Friz (2008). A similar argument holds for the negative tail.

\section{A.5 Proof of Proposition 6.1}

Let $A(t, S ; u)$ be the American put price when the earnings announcement is scheduled at time $T_{e}=$ $u, t<u \leq T$. Our goal is to show that $A(t, S ; u) \geq A(t, S ; l)$, for $t<u \leq l$. W.l.o.g., let $t=0$ and write $A(S ; l) \equiv A(0, S ; l)$. Let $X_{s}=\log \left(S_{s} / S_{0}\right)-1_{\left\{s \geq T_{e}\right\}} Z_{e}$ be the $\log$ stock price excluding the EA jump, and denote by $\left(\mathcal{F}_{s}^{u}\right)_{0 \leq s \leq T}\left(\operatorname{resp} .\left(\mathcal{F}_{s}^{l}\right)_{0 \leq s \leq T}\right)$ the filtration generated by $S$ with $T_{e}=u\left(\right.$ resp. $\left.T_{e}=l\right)$. Notice

\footnotetext{
${ }^{6}$ For the derivation of the Heston m.g.f., see, e.g. del Baño Rollin et al. (2009).
} 
that $\mathcal{F}_{s}^{l}=\mathcal{F}_{s}^{u}$ for $s<u$ or $s \geq l$, and $\mathcal{F}_{s}^{l} \subset \mathcal{F}_{s}^{u}$, for $u \leq s<l$. Therefore, the sets of stopping times w.r.t to $\mathcal{F}^{u}$ and $\mathcal{F}^{l}$, denoted respectively by $\mathcal{T}^{u}$ and $\mathcal{T}^{l}$, satisfy $\mathcal{T}^{l} \subset \mathcal{T}^{u}$. Thus, for any candidate stopping time $\tau \in \mathcal{T}^{l}$, we have

$$
\begin{aligned}
& \mathbb{E}\left\{e^{-r \tau}\left(K-S e^{X_{\tau}+1_{\{\tau \geq u\}} Z_{e}}\right)^{+} \mid \mathcal{F}_{l-}^{l}\right\} \\
& =\mathbb{E}\left\{1_{\{\tau<l\}} e^{-r \tau}\left(K-S e^{X_{\tau}+1_{\{\tau \geq u\}} Z_{e}}\right)^{+} \mid \mathcal{F}_{l-}^{l}\right\}+\mathbb{E}\left\{1_{\{\tau \geq l\}} e^{-r \tau}\left(K-S e^{X_{\tau}+1_{\{\tau \geq u\}} Z_{e}}\right)^{+} \mid \mathcal{F}_{l-}^{l}\right\} \\
& \geq \mathbb{E}\left\{1_{\{\tau<l\}} e^{-r \tau}\left(K-S e^{X_{\tau}}\right)^{+} \mid \mathcal{F}_{l-}^{l}\right\}+\mathbb{E}\left\{1_{\{\tau \geq l\}} e^{-r \tau}\left(K-S e^{X_{\tau}+1_{\{\tau \geq l\}} Z_{e}}\right)^{+} \mid \mathcal{F}_{l-}^{l}\right\} \\
& =\mathbb{E}\left\{e^{-r \tau}\left(K-S e^{X_{\tau}+1_{\{\tau \geq l\}} Z_{e}}\right)^{+} \mid \mathcal{F}_{l-}^{l}\right\} .
\end{aligned}
$$

The inequality holds as follows. In the first term of (A.24), given the information in $\mathcal{F}_{l-}^{l}$ and on $\{\tau<l\}$ the values of $\tau$ and $X_{\tau}$ are known, we apply Jensen's inequality to get the first term in (A.25). In addition, since $\tau \geq l$ implies that $\tau \geq u$, the second terms of (A.24) and (A.25) are equal. The last equality follows from the fact that $1_{\{\tau \geq l\}}=0$ on $\{\tau<l\}$.

In turn, taking expectations in (A.23) and (A.26) and maximizing both sides over $\mathcal{T}^{l}$, we get

$$
A(S ; u) \geq \sup _{\tau \in \mathcal{T} l} \mathbb{E}\left\{e^{-r \tau}\left(K-S e^{X_{\tau}+1_{\{\tau \geq u\}} Z_{e}}\right)^{+}\right\} \geq A(S ; l),
$$

where the first inequality follows from the inclusion $\mathcal{T}^{l} \subset \mathcal{T}^{u}$.

\section{A.6 Barone-Adesi Approximation}

Here we give a sketch of the derivation of the approximation formula (6.7). The arguments are adapted from Barone-Adesi and Whaley (1987) and Kou and Wang (2004). We start by writing

$$
A(t, S)=P_{E}(t, S)+\epsilon(t, S),
$$

where $P_{E}$ is the European put price with an EA jump, and $\epsilon$ is a correction term. Note that $P_{E}$ is computed using the results in Proposition 3.1. In the continuation region, $\epsilon$ must satisfy the same PIDE as that of $P_{E}$ and $A$, namely

$$
r \epsilon(t, S)-\partial_{t} \epsilon(t, S)-\mathcal{L} \epsilon(t, S)=0
$$

where the operator $\mathcal{L}$ is defined in (6.5). The idea of the approximation in Barone-Adesi and Whaley (1987) is to remove the term $\epsilon_{t}$ in (A.27). This involves letting $\epsilon(t, S) \equiv g(z, S) z$, with $z \equiv 1-e^{-r(T-t)}$, substituting in the above PIDE, and ignoring the term $(1-z) g_{z}$. This results in the OIDE

$$
\frac{r}{z} \epsilon(t, S)-\mathcal{L} \epsilon(t, S)=0
$$

While (A.28) holds in the continuation region, in the exercise reason we have $\epsilon(t, S)=K-S-P_{E}(t, S)$. Following Kou and Wang (2004), we consider the ansatz

$$
\epsilon(t, S)= \begin{cases}\gamma_{1}(t) S^{-\beta_{1}}+\gamma_{2}(t) S^{-\beta_{2}} & , \quad S>\alpha(t), \\ K-S-P_{E}(t, S) & , \quad S \leq \alpha(t),\end{cases}
$$

where $\alpha(t)$ is the boundary at time $t$. One can directly verify that the ansatz solves the OIDE (A.28) if $\beta_{1,2}$ are two positive solutions to 6.10 , and if

$$
\frac{K}{\lambda_{2}}-\frac{\alpha(t)}{1+\lambda_{2}}-\int_{-\infty}^{0} P_{E}\left(t, \alpha e^{y}\right) e^{\lambda_{2} y} d y=\gamma_{1}(t) \frac{\alpha^{-\beta_{1}}}{\lambda_{2}-\beta_{1}}+\gamma_{2}(t) \frac{\alpha^{-\beta_{2}}}{\lambda_{2}-\beta_{2}},
$$

In turn, we impose the continuous-fit and smooth-pasting conditions. The first condition yields (6.11) while the second condition, altogether with (A.29), yields the constants $\gamma_{1}$ and $\gamma_{2}$ in (6.8) and (6.9). 


\section{References}

Andersen, L. and Andreasen, J. (2000). Jump diffusion models: volatility smile fitting and numerical methods for pricing. Review of Derivatives Research, 4:231-262.

Barone-Adesi, G. and Whaley, R. (1987). Efficient analytic approximation of American option values. The Journal of Finance, 17(2):301-320.

Barth, M., Johnson, T., and So, C. (2011). Dynamics of earnings announcement news: Evidence from option prices. Working paper.

Bates, D. (1996). Jumps and stochastic volatility: The exchange rate processes implicit in Deutschemark options. Review of Financial Studies, 9(1):69-107.

Benaim, S. and Friz, P. (2008). Smile asymptotics II: models with known moment generating functions. Journal of Applied Probability, 45 (1):1-291.

Benaim, S., Friz, P., and Lee, R. (2012). On the Black-Scholes implied volatility at extreme strikes. In Cont, R., editor, Frontiers in Quantitative Finance: Volatility and Credit Risk Modeling, pages 19-45. Wiley \& Sons.

Bensoussan, A. and Lions, J. (1984). Impulse control and quasivariational inequalities. Gaunthier-Villars.

Billings, M. and Jennings, R. (2011). The option markets anticipation of information content in earnings announcements. Review of Accounting Studies Conference Version, 16:587-619.

Black, F. and Scholes, M. (1973). The pricing of options and corporate liabilities. Journal of Political Economy, 81:637-654.

Broadie, M., Chernov, M., and Johannes, M. (2009). Understanding index option returns. Review of Financial Studies, 22(11):4493-4529.

Carr, P., Geman, H., Madan, D., and Yor, M. (2002). The fine structure of asset returns: An empirical investigation. Journal of Business, 75(2):305-332.

Carr, P. and Madan, D. (1999). Option pricing and the fast Fourier transform. Journal of Computational Finance, $2: 61-73$.

Chatrah, A., Christie-David, R., and Lee, K. (2009). How potent are news reversals?: Evidence from the futures markets. Journal of Futures Market, 29:42-73.

Chordia, T. and Shivakumar, L. (2006). Earnings and price momentum. Journal of Financial Economics, 80(3):627656.

Coleman, T. and Li, Y. (1994). On the convergence of reflective Newton methods for large-scale nonlinear minimization subject to bounds. Mathematical Programming, 67, 2:189-224.

Coleman, T. and Li, Y. (1996). An interior, trust region approach for nonlinear minimization subject to bounds. SIAM Journal on Optimization, 6:418-445.

Cont, R. and Tankov, P. (2002). Calibration of jump-diffusion option-pricing models: a robust non-parametric approach. Working paper.

Cont, R. and Voltchkova, E. (2005). A finite difference scheme for option pricing in jump diffusions and exponential Lévy models. SIAM Journal on Numerical Analysis, 43:1596-1626.

del Baño Rollin, S., Ferreiro-Castilla, A., and Utzet, F. (2009). A new look at the Heston characteristic function. Preprint.

Dennis, J. (1977). Nonlinear least-squares and equations. In Jacobs, D., editor, State of the Art in Numerical Analysis, pages 269-312. Academic Press.

Donders, M. and Vorst, T. (1996). The impact of firm specific news on IVs. Journal of Banking and Finance, $20: 1447-1461$.

Dubinsky, A. and Johannes, M. (2006). Fundamental uncertainty, earning announcements and equity options. Working paper. 
Duffie, D., J. P. and Singleton, K. (2000). Transform analysis and option pricing for affine jump-diffusions. Econometrica, 68:1343-1376.

Isakov, D. and Perignon, C. (2001). Evolution of market uncertainty around earnings announcements. Journal of Banking and Finance, 25:1769-1788.

Jackson, K., Jaimungal, S., and Surkov, V. (2008). Fourier space time-stepping for option pricing with Lévy models. Journal of Computational Finance, 12(2):1-29.

Kou, S. (2002). A jump-diffusion model for option pricing. Management Science, 48:1086-1101.

Kou, S. G. and Wang, H. (2004). Option pricing under a double exponential jump diffusion model. Management Science, 50(9):1178-1192.

Lee, R. (2004). Option pricing by transform methods: extensions, unification, and error control. Journal of Computational Finance, 7:51-86.

Lee, S. and Mykland, P. (2008). Jumps in financial markets: A new nonparametric test and jump dynamics. Review of Financial Studies, 21(6):2535-2563.

Lord, R., Fang, F., Bervoets, F., and Oosterlee, C. W. (2008). A fast and accurate FFT-based method for pricing early-exercise options under Lévy processes. SIAM Journal of Scientific Computing, 30(4):1678-1705.

Madan, D., Carr, P., and Chang, E. (1998). The variance gamma process and option pricing. European Finance Review, 2(8):79-105.

Maheu, J. and McCurdy, T. (2004). News arrival, jump dynamics and volatility components for individual stock returns. Journal of Finance, 59:755-793.

Mehra, A., Kolanovic, M., and Kaplan, B. (2014). Earnings and option volatility monitor. Technical report, J.P. Morgan.

Merton, R. (1973). Theory of rational option pricing. Bell Journal of Economics and Management Science, 4:141-183.

Merton, R. (1976). Option pricing when underlying stock returns are discontinuous. Journal of Financial Economics, 3:125-144.

Øksendal, B. (2003). Stochastic Differential Equations: An Introduction with Applications. Springer.

Patell, J. and Wolfson, M. (1981). The ex ante and ex post price effect of quarterly earnings announcements reflected in option and stock prices. Journal of Accounting Research, 19:434-458.

Patell, J. and Wolfson, M. (1984). The intraday speed of adjustment of stock prices to earnings and dividend announcements. Journal of Financial Economics, 13:223-252.

Piazzesi, M. (2005). Bond yields and the Federal Reserve. Journal of Political Economy, 113:311-344.

Raible, S. (2000). Lévy processes in finance: Theory, numerics, and empirical facts. PhD thesis, Univ. Freiburg.

Rogers, J., Skinner, D., and Van Buskirk, A. (2009). Earnings guidance and market uncertainty. Journal of Accounting and Economics, 48:90-109.

\section{References}

Andersen, L. and Andreasen, J. (2000). Jump diffusion models: volatility smile fitting and numerical methods for pricing. Review of Derivatives Research, 4:231-262.

Barone-Adesi, G. and Whaley, R. (1987). Efficient analytic approximation of American option values. The Journal of Finance, 17(2):301-320.

Barth, M., Johnson, T., and So, C. (2011). Dynamics of earnings announcement news: Evidence from option prices. Working paper. 
Bates, D. (1996). Jumps and stochastic volatility: The exchange rate processes implicit in Deutschemark options. Review of Financial Studies, 9(1):69-107.

Benaim, S. and Friz, P. (2008). Smile asymptotics II: models with known moment generating functions. Journal of Applied Probability, 45 (1):1-291.

Benaim, S., Friz, P., and Lee, R. (2012). On the Black-Scholes implied volatility at extreme strikes. In Cont, R., editor, Frontiers in Quantitative Finance: Volatility and Credit Risk Modeling, pages 19-45. Wiley \& Sons.

Bensoussan, A. and Lions, J. (1984). Impulse control and quasivariational inequalities. Gaunthier-Villars.

Billings, M. and Jennings, R. (2011). The option markets anticipation of information content in earnings announcements. Review of Accounting Studies Conference Version, 16:587-619.

Black, F. and Scholes, M. (1973). The pricing of options and corporate liabilities. Journal of Political Economy, 81:637-654.

Broadie, M., Chernov, M., and Johannes, M. (2009). Understanding index option returns. Review of Financial Studies, 22(11):4493-4529.

Carr, P., Geman, H., Madan, D., and Yor, M. (2002). The fine structure of asset returns: An empirical investigation. Journal of Business, 75(2):305-332.

Carr, P. and Madan, D. (1999). Option pricing and the fast Fourier transform. Journal of Computational Finance, $2: 61-73$.

Chatrah, A., Christie-David, R., and Lee, K. (2009). How potent are news reversals?: Evidence from the futures markets. Journal of Futures Market, 29:42-73.

Chordia, T. and Shivakumar, L. (2006). Earnings and price momentum. Journal of Financial Economics, 80(3):627656.

Coleman, T. and Li, Y. (1994). On the convergence of reflective Newton methods for large-scale nonlinear minimization subject to bounds. Mathematical Programming, 67, 2:189-224.

Coleman, T. and Li, Y. (1996). An interior, trust region approach for nonlinear minimization subject to bounds. SIAM Journal on Optimization, 6:418-445.

Cont, R. and Tankov, P. (2002). Calibration of jump-diffusion option-pricing models: a robust non-parametric approach. Working paper.

Cont, R. and Voltchkova, E. (2005). A finite difference scheme for option pricing in jump diffusions and exponential Lévy models. SIAM Journal on Numerical Analysis, 43:1596-1626.

del Baño Rollin, S., Ferreiro-Castilla, A., and Utzet, F. (2009). A new look at the Heston characteristic function. Preprint.

Dennis, J. (1977). Nonlinear least-squares and equations. In Jacobs, D., editor, State of the Art in Numerical Analysis, pages 269-312. Academic Press.

Donders, M. and Vorst, T. (1996). The impact of firm specific news on IVs. Journal of Banking and Finance, 20:1447-1461.

Dubinsky, A. and Johannes, M. (2006). Fundamental uncertainty, earning announcements and equity options. Working paper.

Duffie, D., J. P. and Singleton, K. (2000). Transform analysis and option pricing for affine jump-diffusions. Econometrica, 68:1343-1376.

Isakov, D. and Perignon, C. (2001). Evolution of market uncertainty around earnings announcements. Journal of Banking and Finance, 25:1769-1788.

Jackson, K., Jaimungal, S., and Surkov, V. (2008). Fourier space time-stepping for option pricing with Lévy models. Journal of Computational Finance, 12(2):1-29.

Kou, S. (2002). A jump-diffusion model for option pricing. Management Science, 48:1086-1101. 
Kou, S. G. and Wang, H. (2004). Option pricing under a double exponential jump diffusion model. Management Science, 50(9):1178-1192.

Lee, R. (2004). Option pricing by transform methods: extensions, unification, and error control. Journal of Computational Finance, 7:51-86.

Lee, S. and Mykland, P. (2008). Jumps in financial markets: A new nonparametric test and jump dynamics. Review of Financial Studies, 21(6):2535-2563.

Lord, R., Fang, F., Bervoets, F., and Oosterlee, C. W. (2008). A fast and accurate FFT-based method for pricing early-exercise options under Lévy processes. SIAM Journal of Scientific Computing, 30(4):1678-1705.

Madan, D., Carr, P., and Chang, E. (1998). The variance gamma process and option pricing. European Finance Review, 2(8):79-105.

Maheu, J. and McCurdy, T. (2004). News arrival, jump dynamics and volatility components for individual stock returns. Journal of Finance, 59:755-793.

Mehra, A., Kolanovic, M., and Kaplan, B. (2014). Earnings and option volatility monitor. Technical report, J.P. Morgan.

Merton, R. (1973). Theory of rational option pricing. Bell Journal of Economics and Management Science, $4: 141-183$.

Merton, R. (1976). Option pricing when underlying stock returns are discontinuous. Journal of Financial Economics, 3:125-144.

Øksendal, B. (2003). Stochastic Differential Equations: An Introduction with Applications. Springer.

Patell, J. and Wolfson, M. (1981). The ex ante and ex post price effect of quarterly earnings announcements reflected in option and stock prices. Journal of Accounting Research, 19:434-458.

Patell, J. and Wolfson, M. (1984). The intraday speed of adjustment of stock prices to earnings and dividend announcements. Journal of Financial Economics, 13:223-252.

Piazzesi, M. (2005). Bond yields and the Federal Reserve. Journal of Political Economy, 113:311-344.

Raible, S. (2000). Lévy processes in finance: Theory, numerics, and empirical facts. PhD thesis, Univ. Freiburg.

Rogers, J., Skinner, D., and Van Buskirk, A. (2009). Earnings guidance and market uncertainty. Journal of Accounting and Economics, 48:90-109. 